\title{
Total Synthesis of Thiostrepton. Assembly of Key Building Blocks and Completion of the Synthesis.
}

\author{
K. C. Nicolaou, * Mark Zak, Brian S. Safina, Anthony A. Estrada, Sang Hyup Lee, Marta
}

Nevalainen.

Contribution from the Department of Chemistry and The Skaggs Institute for Chemical Biology, The Scripps Research Institute, 10550 North Torrey Pines Road, La Jolla, California 92037, and Department of Chemistry and Biochemistry, University of California, San Diego, 9500 Gilman Drive, La Jolla, California 92093.

Corresponding author e-mail: kcn@scripps.edu

\section{Supporting Information}

\section{Experimental Data for Compounds}

General Procedures. All reactions were carried out under an argon atmosphere with dry solvents under anhydrous conditions, unless otherwise noted. Dry tetrahydrofuran (THF), toluene, benzene, diethyl ether $\left(\mathrm{Et}_{2} \mathrm{O}\right)$, ethylene glycol dimethyl ether (DME), and methylene chloride $\left(\mathrm{CH}_{2} \mathrm{Cl}_{2}\right)$ were obtained by passing commercially available pre-dried, oxygen-free formulations through activated alumina columns. Yields refer to chromatographically and spectroscopically ( ${ }^{1} \mathrm{H}$ NMR) homogeneous materials, unless otherwise stated. Reagents were 
purchased at the highest commercial quality and used without further purification, unless otherwise stated. Reactions were monitored by thin-layer chromatography (TLC) carried out on $0.25 \mathrm{~mm}$ E. Merck silica gel plates (60F-254) using UV light as visualizing agent and an ethanolic solution of phosphomolybdic acid and cerium sulfate, and heat as developing agents. E. Merck silica gel (60, particle size $0.040-0.063 \mathrm{~mm})$ was used for flash column chromatography. Preparative thin-layer chromatography (PTLC) separations were carried out on 0.25 or $0.50 \mathrm{~mm}$ E. Merck silica gel plates (60F-254). NMR spectra were recorded on Bruker DRX-600, DRX500, AMX-500 or AMX-400 instruments and calibrated using residual undeuterated solvent as an internal reference. The following abbreviations were used to explain the multiplicities: $\mathrm{s}=$ singlet, $\mathrm{d}=$ doublet, $\mathrm{t}=$ triplet, $\mathrm{q}=$ quartet, $\mathrm{m}=$ multiplet, quin $=$ quintuplet, $\mathrm{sext}=$ sextet, $\mathrm{sep}=$ septet, $\mathrm{br}=$ broad. IR spectra were recorded on a Perkin-Elmer 1600 series FT-IR spectrometer. Electrospray ionization (ESI) mass spectrometry (MS) experiments were performed on an API 100 Perkin Elmer SCIEX single quadrupole mass spectrometer at 4000V emitter voltage. Highresolution mass spectra (HRMS) were recorded on a VG ZAB-ZSE mass spectrometer using MALDI (matrix-assisted laser-desorption ionization) or ESI (electrospray ionization).

\section{$N$-Alloc dehydropiperidine 6 (Scheme 1):}

To a solution of the less polar $5 R, 6 S$ amine isomer $\mathbf{2}^{1}(1.26 \mathrm{~g}, 1.68 \mathrm{mmol})$ in THF $(26 \mathrm{~mL})$ at 25 ${ }^{\circ} \mathrm{C}$ was added $i-\mathrm{Pr}_{2} \mathrm{NEt}(2.9 \mathrm{~mL}, 16.9 \mathrm{mmol})$, allyl chloroformate $(0.88 \mathrm{~mL}, 8.40 \mathrm{mmol})$, and $4-$

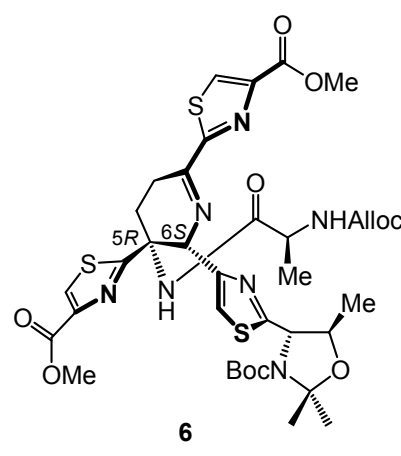
DMAP (21 mg, $0.17 \mathrm{mmol}$ ). The reaction mixture was stirred for $3 \mathrm{~h}$ at $25{ }^{\circ} \mathrm{C}$ and then diluted with ethyl acetate $(200 \mathrm{~mL})$. The organic layer was washed with aqueous $0.05 \mathrm{M} \mathrm{KHSO}_{4}$ solution, saturated aqueous $\mathrm{NaHCO}_{3}$ solution, and brine $(50 \mathrm{~mL}$ each), then dried $\left(\mathrm{Na}_{2} \mathrm{SO}_{4}\right)$ and concentrated. The crude residue was purified by flash column chromatography (silica gel, hexanes:EtOAc, 60:40 $\rightarrow$ 30:70) to give $N$-Alloc dehydropiperidine $6(1.28 \mathrm{~g}, 92 \%)$ as a yellow foam.

6: $R_{\mathrm{f}}=0.43$ (silica gel, hexanes:EtOAc, $\left.2: 8\right) ;[\alpha]_{\mathrm{D}}^{32}=+14.2\left(\mathrm{CHCl}_{3}, c=1.00\right)$; IR (film) $v_{\max }$ 3318, 3096, 2966, 2919, 1719, 1701, 1502, 1478, 1367, 1237, 1214, 1132, 1096, 991, $773 \mathrm{~cm}^{-1}$; 
${ }^{1} \mathrm{H}$ NMR $\left(500 \mathrm{MHz}, \mathrm{CD}_{3} \mathrm{CN}, 70{ }^{\circ} \mathrm{C}\right) \delta 8.27(\mathrm{~s}, 1 \mathrm{H}), 8.04(\mathrm{~s}, 1 \mathrm{H}), 7.13(\mathrm{~s}, 1 \mathrm{H}), 5.84-5.75(\mathrm{~m}$, $1 \mathrm{H}), 5.68$ (br s, $1 \mathrm{H}), 5.35$ (br s, $1 \mathrm{H}), 5.20$ (dq, $J=17.3,1.8 \mathrm{~Hz}, 1 \mathrm{H}), 5.11$ (dq, $J=11.6,1.8$ $\mathrm{Hz}, 1 \mathrm{H}), 4.75$ (d, $J=5.8 \mathrm{~Hz}, 1 \mathrm{H}), 4.30$ (dd, $J=13.2,6.3 \mathrm{~Hz}, 1 \mathrm{H}), 4.31-4.17$ (br, $2 \mathrm{H}), 3.90$ (s, $3 \mathrm{H}), 3.85$ (s, $3 \mathrm{H}), 3.41$ (ddd, $J=13.9,7.0,2.2 \mathrm{~Hz}, 1 \mathrm{H}$ ), 3.14 (ddt, $J=19.8,6.3,2.2 \mathrm{~Hz}, 1$ H), $2.97-2.86(\mathrm{~m}, 1 \mathrm{H}), 2.72-2.63(\mathrm{~m}, 1 \mathrm{H}), 1.64(\mathrm{~s}, 3 \mathrm{H}), 1.59$ (s, $3 \mathrm{H}), 1.39$ (d, $J=6.3 \mathrm{~Hz}, 3$ $\mathrm{H}), 1.33(\mathrm{br}, 9 \mathrm{H}), 1.31(\mathrm{~d}, J=6.9 \mathrm{~Hz}, 3 \mathrm{H}) ;{ }^{13} \mathrm{C} \mathrm{NMR}\left(125 \mathrm{MHz}, \mathrm{CD}_{3} \mathrm{CN}, 70{ }^{\circ} \mathrm{C}\right) \delta 176.7$, 174.3, 173.9, 170.7, 164.7, 162.7, 157.0, 153.5, 148.8, 147.8, 134.4, 131.9, 129.1, 120.3, 96.1, $81.8,78.7,67.7,67.2,66.5,61.1,53.4,52.9,52.8,28.8,28.4,26.0,19.1,18.5$; HRMS (ESITOF) calcd for $\mathrm{C}_{36} \mathrm{H}_{46} \mathrm{~N}_{7} \mathrm{O}_{10} \mathrm{~S}_{3}{ }^{+}\left[\mathrm{M}+\mathrm{H}^{+}\right]$832.2463, found 832.2459.

\section{Seco diester 8 (Scheme 1):}

To $N$-Alloc dehydropiperidine $6(1.09 \mathrm{~g}, 1.31 \mathrm{mmol})$ in $\mathrm{CH}_{2} \mathrm{Cl}_{2}(42 \mathrm{~mL})$ was added TFA (42 $\mathrm{mL}$ ) at $0{ }^{\circ} \mathrm{C}$. After stirring for $10 \mathrm{~min}$ at $0{ }^{\circ} \mathrm{C}$, the reaction mixture was brought to $25{ }^{\circ} \mathrm{C}$ and

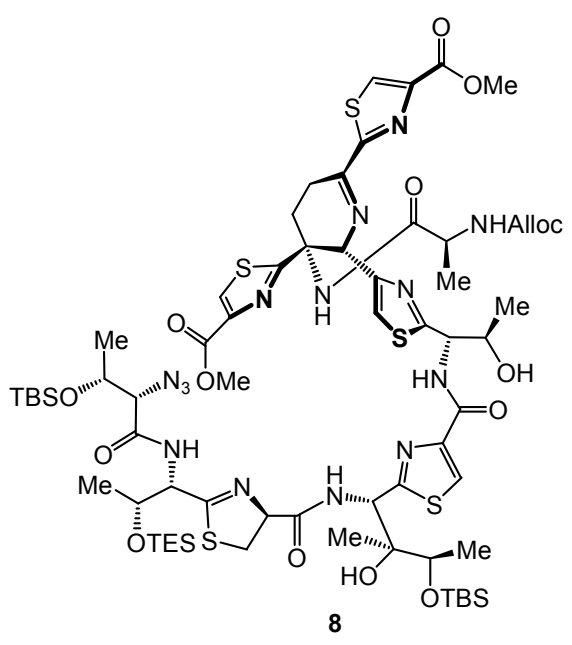
stirring was continued for a further $45 \mathrm{~min}$. The reaction mixture was then concentrated and saturated aqueous $\mathrm{NaHCO}_{3}$ solution $(30 \mathrm{~mL})$ was added. The aqueous layer was extracted with $\mathrm{CH}_{2} \mathrm{Cl}_{2}(3 \times 30 \mathrm{~mL})$, and the combined organic layers were then washed with brine $(30 \mathrm{~mL})$, dried $\left(\mathrm{Na}_{2} \mathrm{SO}_{4}\right)$, and concentrated to give amino alcohol 7 which was used immediately and without further purification in the next step. The crude carboxylic acid $\mathbf{3}^{1}$ and amino alcohol 7 were combined in a flask and dissolved in DMF $(8.3 \mathrm{~mL})$. The resulting pale yellow solution was cooled to $0{ }^{\circ} \mathrm{C}$ and then $i$-Pr 2 NEt (0.69 mL, $\left.3.39 \mathrm{mmol}\right)$, HOAt (214 mg, $1.57 \mathrm{mmol}$ ), and HATU (597 mg, $1.57 \mathrm{mmol}$ ) were added. The reaction mixture was stirred for $30 \mathrm{~min}$ at $0{ }^{\circ} \mathrm{C}$, and then diluted with EtOAc $(50 \mathrm{~mL})$. The organic layer was washed with aqueous $0.01 \mathrm{M} \mathrm{KHSO}_{4}$ solution, saturated aqueous $\mathrm{NaHCO}_{3}$ solution, and brine $\left(20 \mathrm{~mL}\right.$ each), then dried $\left(\mathrm{Na}_{2} \mathrm{SO}_{4}\right)$ and concentrated. The residue was purified by flash column chromatography (silica gel, hexanes:EtOAc, 3:7) to give seco diester $8(1.51 \mathrm{~g}, 73 \%$ over 2 steps from 6) as a white foam.

8: $R_{\mathrm{f}}=0.42$ (silica gel, hexanes:EtOAc, $\left.3: 7\right) ;[\alpha]_{\mathrm{D}}^{32}=+15.6\left(\mathrm{CHCl}_{3}, c=1.00\right)$; IR (film) $v_{\max }$ 3387, 2953, 2837, 2112, 1737, 1677, 1508, 1247, 1101, 992, 843, $778 \mathrm{~cm}^{-1} ;{ }^{1} \mathrm{H}$ NMR (600 MHz, 
$\left.\mathrm{CD}_{3} \mathrm{CN}, 70{ }^{\circ} \mathrm{C}\right) \delta 8.27($ br s, $1 \mathrm{H}), 8.26(\mathrm{~s}, 1 \mathrm{H}), 8.23(\mathrm{~d}, J=8.8 \mathrm{~Hz}, 1 \mathrm{H}), 8.03(\mathrm{~s}, 1 \mathrm{H}), 7.56(\mathrm{~d}$, $J=8.3 \mathrm{~Hz}, 1 \mathrm{H}), 7.43$ (d, $J=8.3 \mathrm{~Hz}, 1 \mathrm{H}), 6.99$ (s, $1 \mathrm{H}), 6.46-6.41$ (br, $1 \mathrm{H}), 5.77$ - 5.71 (m, 1 H), $5.37(\mathrm{~d}, J=8.3 \mathrm{~Hz}, 1 \mathrm{H}), 5.34$ (t, $J=1.3 \mathrm{~Hz}, 1 \mathrm{H}), 5.29(\mathrm{dd}, J=9.2,3.1 \mathrm{~Hz}, 1 \mathrm{H}), 5.17(\mathrm{~d}, J$ $=17.1 \mathrm{~Hz}, 1 \mathrm{H}), 5.07(\mathrm{~d}, J=10.6 \mathrm{~Hz}, 1 \mathrm{H}), 5.02$ (t, $J=8.8 \mathrm{~Hz}, 1 \mathrm{H}), 4.72-4.69$ (br, $1 \mathrm{H}), 4.61$ $(\mathrm{d}, J=8.3 \mathrm{~Hz}, 1 \mathrm{H}), 4.44-4.36(\mathrm{~m}, 2 \mathrm{H}), 4.26(\mathrm{dd}, J=10.4,6.8 \mathrm{~Hz}, 1 \mathrm{H}), 4.14-4.11(\mathrm{br}, 1 \mathrm{H})$, 4.03 (q, $J=6.1 \mathrm{~Hz}, 1 \mathrm{H}), 3.90$ (s, $3 \mathrm{H}), 3.86$ (s, $3 \mathrm{H}), 3.54$ (dd, $J=11.0,9.2 \mathrm{~Hz}, 1 \mathrm{H}), 3.49$ (dd, $J$ $=11.4,7.8 \mathrm{~Hz}, 1 \mathrm{H}), 3.37(\mathrm{ddd}, J=13.6,6.6,1.4 \mathrm{~Hz}, 1 \mathrm{H}), 3.14-3.10(\mathrm{~m}, 1 \mathrm{H}), 2.92-2.83(\mathrm{~m}$, $1 \mathrm{H}), 2.70-2.65(\mathrm{~m}, 1 \mathrm{H}), 1.34(\mathrm{~d}, J=6.5 \mathrm{~Hz}, 3 \mathrm{H}), 1.30(\mathrm{~d}, J=7.4 \mathrm{~Hz}, 3 \mathrm{H}), 1.29(\mathrm{~d}, J=6.5$ $\mathrm{Hz}, 3 \mathrm{H}), 1.24(\mathrm{~d}, J=6.1 \mathrm{~Hz}, 3 \mathrm{H}), 1.23$ (d, $J=6.6 \mathrm{~Hz}, 3 \mathrm{H}), 1.03$ (s, $3 \mathrm{H}), 0.97$ (t, $J=7.9 \mathrm{~Hz}, 9$ H), 0.94 (s, 9 H), 0.91 (s, 9 H), 0.62 (dq, J= 7.9, 4.0 Hz, 6 H), 0.12 (s, 3 H), 0.11 (s, 3 H), 0.09 $(\mathrm{s}, 3 \mathrm{H}), 0.08(\mathrm{~s}, 3 \mathrm{H}) ;{ }^{13} \mathrm{C} \mathrm{NMR}\left(125 \mathrm{MHz}, \mathrm{CD}_{3} \mathrm{CN}, 70{ }^{\circ} \mathrm{C}\right) \delta 176.8,175.2,174.7,173.8,173.0$, $172.0,171.7,170.7,170.3,164.4,162.7,162.7,162.6,157.1,153.9,149.8,148.8,147.7,134.3$, $131.9,129.1,125.8,119.8,79.3,77.1,71.6,71.0,70.6,70.4,70.2,69.9,69.0,67.9,66.4,61.1$, $60.9,59.8,58.3,57.5,53.6,52.9,52.8,37.3,28.2,26.7,26.5,25.7,21.9,21.5,21.1,19.5,19.3$, 18.9, 18.8, 18.4, 18.3, 7.2, 6.0, -3.2, -4.0, -4.2, -4.4; HRMS (ESI-TOF) calcd for $\mathrm{C}_{66} \mathrm{H}_{103} \mathrm{~N}_{14} \mathrm{O}_{15} \mathrm{~S}_{5} \mathrm{Si}_{3}^{+}\left[\mathrm{M}+\mathrm{H}^{+}\right]$1575.5633, found 1575.5609 .

\section{Thiazoline macrocycle 11 (Scheme 1):}

Trimethyltin hydroxide $(69 \mathrm{mg}, 0.38 \mathrm{mmol})$ was added to seco diester 8 (300 $\mathrm{mg}, 0.19 \mathrm{mmol})$ in 1,2-dichloroethane $(4.0 \mathrm{~mL})$. The reaction mixture was heated at $50{ }^{\circ} \mathrm{C}$ for $4 \mathrm{~h}$ during which

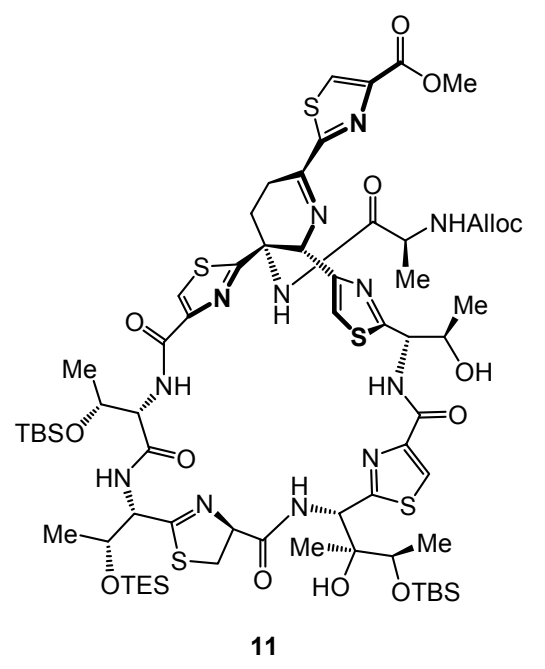

THF:water $(0.50 \mathrm{~mL})$ at $0{ }^{\circ} \mathrm{C}$ was added trimethylphosphine $(1.0 \mathrm{M}$ solution in $\mathrm{THF}, 0.30 \mathrm{~mL}$, 
$0.30 \mathrm{mmol}$ ). The reaction mixture was stirred for $1 \mathrm{~h}$ at $0{ }^{\circ} \mathrm{C}$ and then a further $0.30 \mathrm{~mL}$ of 1.0 $\mathrm{M} \mathrm{Me}_{3} \mathrm{P}$ solution in THF $(0.30 \mathrm{mmol})$ was added. Stirring was continued for $15 \mathrm{~min}$ at $0{ }^{\circ} \mathrm{C}$, and then air was bubbled through the mixture for $15 \mathrm{~min}$. Toluene $(5 \mathrm{~mL})$ was then added and the mixture was dried $\left(\mathrm{Na}_{2} \mathrm{SO}_{4}\right)$ and concentrated to give crude regioisomeric amino acids $\mathbf{1 0}$ and 10' which were used without purification in the next step. To the crude mixture of chromatographically inseparable amino acids 10 and $\mathbf{1 0}$ ' was added DMF (50 mL, $0.002 \mathrm{M}$ ), followed by HATU (188 $\mathrm{mg}, 0.50 \mathrm{mmol})$, HOAt $(67 \mathrm{mg}, 0.50 \mathrm{mmol})$, and $i-\mathrm{Pr}_{2} \mathrm{NEt}(0.11 \mathrm{~mL}$, $0.60 \mathrm{mmol}$ ). The reaction mixture was stirred for $65 \mathrm{~h}$ at $25^{\circ} \mathrm{C}$, and then concentrated. The residue was taken up in EtOAc $(50 \mathrm{~mL})$ and the organic layer was washed with $0.01 \mathrm{M}$ aqueous $\mathrm{KHSO}_{4}$ solution, water, saturated aqueous $\mathrm{NaHCO}_{3}$ solution, and brine $(20 \mathrm{~mL}$ each), then dried $\left(\mathrm{Na}_{2} \mathrm{SO}_{4}\right)$ and concentrated. The residue was purified first by flash column chromatography (silica gel, toluene:EtOAc, 1:1), and the resulting impure product was then repurified by preparative TLC (silica gel, hexanes:EtOAc, 4:6, eluted $2 \times$ ) to give thiazoline macrocycle 11 (48 $\mathrm{mg}, 32 \%$ over 2 steps from mixture of monoacids 9 and $\mathbf{9}^{\prime}$ ) as a yellow foam.

11: $R_{\mathrm{f}}=0.37$ (silica gel, hexanes:EtOAc, $\left.3: 7\right) ;[\alpha]_{\mathrm{D}}{ }^{32}=+16.9\left(\mathrm{CHCl}_{3}, c=1.00\right.$ ); IR (film) $v_{\max }$ 3394, 2927, 1718, 1670, 1528, 1483, 1256, 1101, 833, $757 \mathrm{~cm}^{-1} ;{ }^{1} \mathrm{H}$ NMR $\left(500 \mathrm{MHz}, \mathrm{CD}_{3} \mathrm{CN}\right.$, $\left.70{ }^{\circ} \mathrm{C}\right) \delta 8.30(\mathrm{~s}, 1 \mathrm{H}), 8.16(\mathrm{~s}, 1 \mathrm{H}), 7.87(\mathrm{~d}, J=10.0 \mathrm{~Hz}, 1 \mathrm{H}), 7.77(\mathrm{~d}, J=8.4 \mathrm{~Hz}, 1 \mathrm{H}), 7.37$ (s, $2 \mathrm{H}), 5.95-5.90(\mathrm{br}, 1 \mathrm{H}), 5.80-5.71(\mathrm{~m}, 1 \mathrm{H}), 5.57(\mathrm{~d}, J=8.8 \mathrm{~Hz}, 1 \mathrm{H}), 5.37-5.33(\mathrm{~m}, 1$ H), $5.27-5.22(\mathrm{~m}, 1 \mathrm{H}), 5.18$ (dq, $J=17.3,1.5 \mathrm{~Hz}, 1 \mathrm{H}), 5.08$ (dq, $J=10.7,1.5 \mathrm{~Hz}, 1 \mathrm{H}), 4.98$ (dt, $J=9.2,1.9 \mathrm{~Hz}, 1 \mathrm{H}), 4.83$ (d, $J=8.8 \mathrm{~Hz}, 1 \mathrm{H}), 4.73-4.69$ (br, $1 \mathrm{H}), 4.64-4.59$ (br, $1 \mathrm{H}$ ), 4.30 - 4.23 (br, $3 \mathrm{H}$ ), 4.10 (q, $J=6.3 \mathrm{~Hz}, 1 \mathrm{H}$ ), 3.95 (quin, $J=7.0 \mathrm{~Hz}, 1 \mathrm{H}$ ), 3.64 (dd, $J=13.2$, $7.4 \mathrm{~Hz}, 1 \mathrm{H}), 3.57$ (d, $J=9.2 \mathrm{~Hz}, 2 \mathrm{H}), 3.24$ (d, $J=16.5 \mathrm{~Hz}, 1 \mathrm{H}), 2.92-2.83(\mathrm{~m}, 1 \mathrm{H}), 2.49-$ $2.42(\mathrm{~m}, 1 \mathrm{H}), 1.32$ (d, $J=6.2 \mathrm{~Hz}, 3 \mathrm{H}), 1.24$ (d, $J=6.3 \mathrm{~Hz}, 3 \mathrm{H}), 1.23$ (d, $J=7.0 \mathrm{~Hz}, 3 \mathrm{H}), 1.21$ $(\mathrm{d}, J=6.6 \mathrm{~Hz}, 3 \mathrm{H}), 1.10(\mathrm{~d}, J=6.3 \mathrm{~Hz}, 3 \mathrm{H}), 1.05(\mathrm{~s}, 3 \mathrm{H}), 0.98(\mathrm{~s}, 9 \mathrm{H}), 0.96(\mathrm{t}, J=8.1 \mathrm{~Hz}, 9$ H), 0.95 (s, 9 H), 0.64 (q, J = 7.7 Hz, 6 H), 0.20 (s, 3 H), 0.19 (s, 3 H), 0.12 (s, 3 H), 0.09 (s, 3 $\mathrm{H}) ;{ }^{13} \mathrm{C}$ NMR $\left(150 \mathrm{MHz}, \mathrm{CD}_{3} \mathrm{CN}, 70{ }^{\circ} \mathrm{C}\right) \delta 174.2,174.1,171.1,170.8,170.1,164.0,162.8$, 162.2, 162.0, 157.2, 156.0, 150.6, 150.1, 149.0, 134.3, 132.1, 126.3, 124.6, 120.2, 79.6, 78.0, $70.8,70.7,69.7,69.6,66.6,59.5,58.9,58.1,53.3,53.0,36.7,32.9,30.6,30.4,30.3,30.2,30.1$, $29.2,27.4,26.8,25.6,21.0,20.4,19.5,19.0,18.9,18.7,18.3,7.5,6.1,-2.6,-3.9,-4.2$; HRMS (ESI-TOF) calcd for $\mathrm{C}_{65} \mathrm{H}_{101} \mathrm{~N}_{12} \mathrm{O}_{14} \mathrm{~S}_{5} \mathrm{Si}_{3}{ }^{+}\left[\mathrm{M}+\mathrm{H}^{+}\right]$1517.5466, found 1517.5432. 


\section{Bis-phenylselenium thiazoline macrocycle 13 (Scheme 2):}

To thiazoline macrocycle 11 (40 mg, $0.027 \mathrm{mmol})$ in 1,2-dicholorethane $(0.40 \mathrm{mmol})$ was added trimethyltin hydroxide ( $48 \mathrm{mg}, 0.26 \mathrm{mmol}$ ). The reaction mixture was heated at $60{ }^{\circ} \mathrm{C}$ for $2.5 \mathrm{~h}$

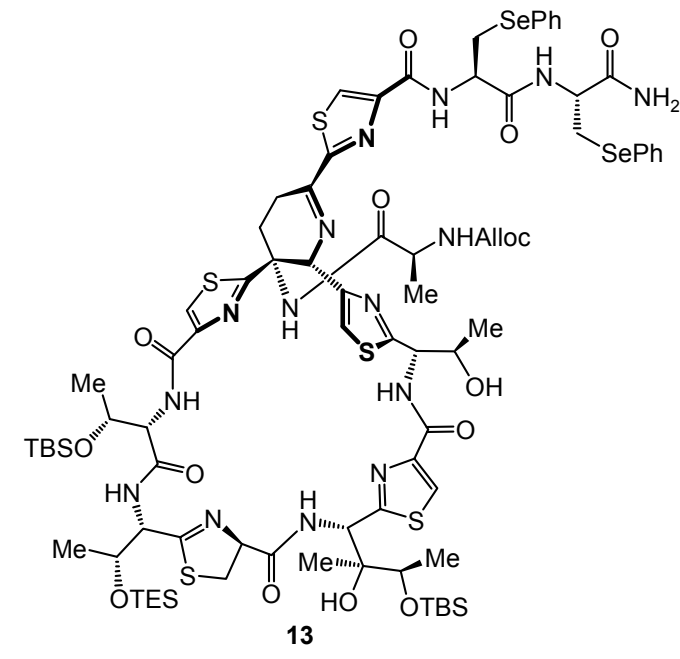
and then concentrated. The residue was taken up in EtOAc $(15 \mathrm{~mL})$ and washed with aqueous $0.01 \mathrm{M}$ $\mathrm{KHSO}_{4}$ solution $(3 \times 5 \mathrm{~mL})$, and water $(5 \mathrm{~mL})$, then dried $\left(\mathrm{Na}_{2} \mathrm{SO}_{4}\right)$ and concentrated to give carboxylic acid $12(40 \mathrm{mg}, 100 \%)$ as a yellow foam, which was used immediately and without further purification in the next step. To carboxylic acid $\mathbf{1 2}(40 \mathrm{mg}, 0.027$ mmol) in DMF $(0.33 \mathrm{~mL})$ at $0{ }^{\circ} \mathrm{C}$ was added bisphenylselenium amine 4 (25 $\mathrm{mg}, 0.051 \mathrm{mmol})$, followed by HOAt ( $4.4 \mathrm{mg}, 0.032 \mathrm{mmol}$ ), HATU (12.0 $\mathrm{mg}, 0.032 \mathrm{mmol})$, and $i-\operatorname{Pr}_{2} \mathrm{NEt}(14 \mu \mathrm{L}, 0.081 \mathrm{mmol})$. The reaction mixture was stirred at $0{ }^{\circ} \mathrm{C}$ for $1.5 \mathrm{~h}$ and then at $25^{\circ} \mathrm{C}$ for $30 \mathrm{~min}$. The reaction mixture was diluted with EtOAc $(5$ $\mathrm{mL})$ and the organic layer was washed with $0.01 \mathrm{M} \mathrm{KHSO}_{4}$ solution $(2 \times 1.5 \mathrm{~mL})$, water $(1.5$ $\mathrm{mL})$, and brine $(1.5 \mathrm{~mL})$, then dried $\left(\mathrm{Na}_{2} \mathrm{SO}_{4}\right)$ and concentrated. The residue was purified by preparative TLC (silica gel, $\mathrm{CH}_{2} \mathrm{Cl}_{2}: \mathrm{MeOH}, 92.5: 7.5$ ) to give bis-phenylselenium thiazoline macrocycle 13 (42 $\mathrm{mg}, 83 \%$ ) as a yellow foam.

13: $R_{\mathrm{f}}=0.40$ (silica gel, $\left.\mathrm{CH}_{2} \mathrm{Cl}_{2}: \mathrm{MeOH}, 93: 7\right) ;[\alpha]_{\mathrm{D}}^{32}=-14.1\left(\mathrm{CHCl}_{3}, c=1.00\right)$; IR (film) $v_{\max }$ 3398, 2927, 2860, 1664, 1529, 1482, 1384, 1256, 1100, 834, $778 \mathrm{~cm}^{-1}$; ${ }^{1} \mathrm{H}$ NMR $(600 \mathrm{MHz}$, $\left.\mathrm{CD}_{3} \mathrm{CN}, 70{ }^{\circ} \mathrm{C}\right) \delta 8.16(\mathrm{~s}, 1 \mathrm{H}), 8.03(\mathrm{~d}, J=7.0 \mathrm{~Hz}, 1 \mathrm{H}), 7.90(\mathrm{~d}, J=9.2 \mathrm{~Hz}, 1 \mathrm{H}), 7.80-7.57$ (m, 2 H), 7.56 - 7.52 (br, 1 H), 7.50 - 7.49 (m, 2 H), 7.39 (s, 1 H), 7.28 - 7.27 (m, 4 H), 7.24 $7.23(\mathrm{~m}, 3 \mathrm{H}), 5.93(\mathrm{br} \mathrm{s}, 1 \mathrm{H}), 5.79-5.75(\mathrm{~m}, 1 \mathrm{H}), 5.58(\mathrm{~d}, J=9.2 \mathrm{~Hz}, 1 \mathrm{H}), 5.39-5.38(\mathrm{~m}, 1$ H), 5.24 (br s, $1 \mathrm{H}), 5.20$ (dd, $J=17.1,1.3 \mathrm{~Hz}, 1 \mathrm{H}), 5.09$ (dd, $J=10.6,1.1 \mathrm{~Hz}, 1 \mathrm{H}), 5.00(\mathrm{dt}, J$ = 9.2, 1.4 Hz, $1 \mathrm{H}), 4.86-4.84(\mathrm{~m}, 1 \mathrm{H}), 4.72-4.65$ (m, $2 \mathrm{H}), 4.60$ (br s, $1 \mathrm{H}), 4.52-4.48$ (m, 2 H), $4.32-4.25(\mathrm{~m}, 4 \mathrm{H}), 4.12(\mathrm{q}, J=6.1 \mathrm{~Hz}, 1 \mathrm{H}), 4.00$ (quin, J = 6.6 Hz, $1 \mathrm{H}$ ), $3.69-3.64(\mathrm{~m}, 1$ H), 3.58 (d, $J=9.2 \mathrm{~Hz}, 2 \mathrm{H}$ ), $3.44-3.19$ (m, $6 \mathrm{H}$ ), 2.89 (br s, $1 \mathrm{H}), 2.46$ (br s, $1 \mathrm{H}$ ), 1.33 (d, $J=$ $6.1 \mathrm{~Hz}, 3 \mathrm{H}), 1.25(\mathrm{~d}, J=6.6 \mathrm{~Hz}, 3 \mathrm{H}), 1.24(\mathrm{~d}, J=6.0 \mathrm{~Hz}, 3 \mathrm{H}), 1.22(\mathrm{~d}, J=6.6 \mathrm{~Hz}, 3 \mathrm{H}), 1.10$ $(\mathrm{d}, J=6.1 \mathrm{~Hz}, 3 \mathrm{H}), 1.05$ (s, $3 \mathrm{H}), 0.98$ (s, $9 \mathrm{H}), 0.97-0.94$ (m, 9 H), 0.95 (s, $9 \mathrm{H}), 0.66$ (q, $J=$ $7.9 \mathrm{~Hz}, 6 \mathrm{H}), 0.20$ (s, $3 \mathrm{H}), 0.19$ (s, $3 \mathrm{H}), 0.12$ (s, $3 \mathrm{H}), 0.089$ (s, $3 \mathrm{H}) ;{ }^{13} \mathrm{C}$ NMR $(150 \mathrm{MHz}$, 
dioxane- $\left.d_{8}, 70{ }^{\circ} \mathrm{C}\right) \delta 173.6,173.0,172.0,170.6,169.7,169.2,161.1,151.2,149.6,133.8,133.2$, 133.1, 131.0, 130.8, 129.7, 129.6, 127.6, 127.4, 119.4, 117.8, 71.1, 66.1, 61.6, 54.2, 53.8, 53.6, $52.1,46.6,33.8,32.3,30.1,29.9,29.8,29.7,29.6,29.5,28.9,26.3,26.2,25.4,25.2,23.0,18.4$, 18.4, 17.8, 14.6, 14.0, 6.9, 5.4, -5.0; HRMS (ESI-TOF) calcd for $\mathrm{C}_{82} \mathrm{H}_{117} \mathrm{~N}_{15} \mathrm{O}_{15} \mathrm{~S}_{5} \mathrm{Se}_{2} \mathrm{Si}_{3} \mathrm{Na}^{+}[\mathrm{M}$ $\left.+\mathrm{Na}^{+}\right]$1978.4987, found 1978.4973 .

\section{Bis-phenylselenium seco compound 15 (Scheme 2):}

To carboxylic acid $14^{1}(9.1 \mathrm{mg}, 0.012 \mathrm{mmol})$ at $25{ }^{\circ} \mathrm{C}$ was added a solution of 2,4,6trichlorobenzoyl chloride $(3.7 \mu \mathrm{L}, 0.023 \mathrm{mmol})$ and $\mathrm{Et}_{3} \mathrm{~N}(0.010 \mathrm{~mL}, 0.071 \mathrm{mmol})$ in toluene

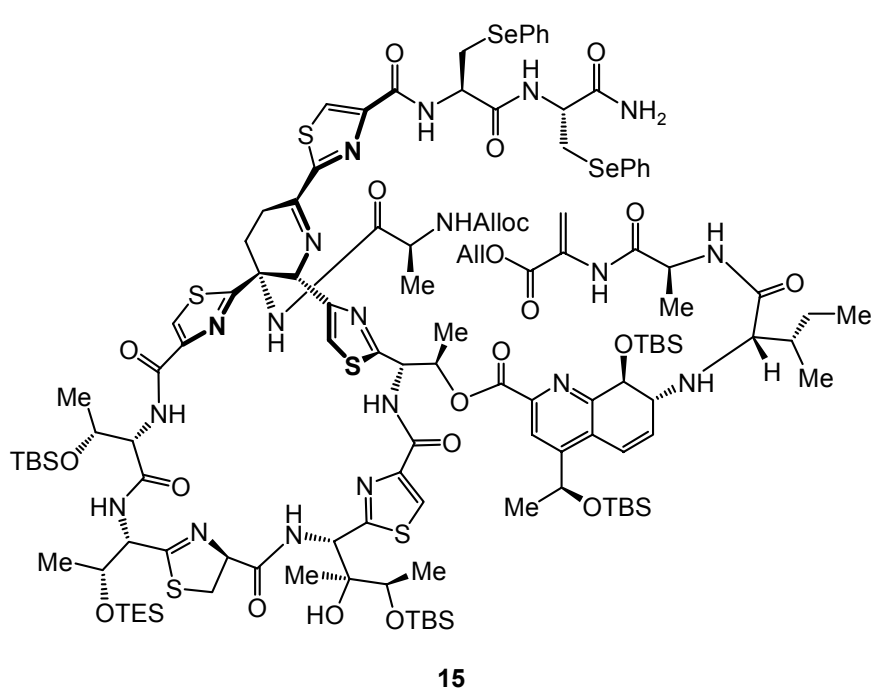
$(0.10 \mathrm{~mL})$. The reaction mixture was stirred for $16 \mathrm{~h}$ at $25{ }^{\circ} \mathrm{C}$ and then added to bis-phenylselenium thiazoline macrocycle 13 (11 $\mathrm{mg}, 0.0060 \mathrm{mmol})$ and 4-DMAP (11 $\mathrm{mg}, 0.090 \mathrm{mmol})$ and stirred for 35 min at $25^{\circ} \mathrm{C}$. The reaction mixture was then purified directly by preparative TLC (silica gel, EtOAc) to give bisphenylselenium seco compound $\mathbf{1 5}$ as a yellow oil (12.8 $\mathrm{mg}, 84 \%)$.

15: $R_{\mathrm{f}}=0.38$ (silica gel, $\left.\mathrm{CH}_{2} \mathrm{Cl}_{2}: \mathrm{MeOH}, 93: 7\right) ;[\alpha]_{\mathrm{D}}{ }^{32}=-50.0\left(\mathrm{CHCl}_{3}, c=0.70\right)$; IR (film) $v_{\max }$ 3366, 2926, 2854, 1718, 1684, 1654, 1541, 1522, 1490, 1474, 1376, 1252, 1098, 853, $779 \mathrm{~cm}^{-1}$; ${ }^{1} \mathrm{H}$ NMR $\left(500 \mathrm{MHz}, \mathrm{CD}_{3} \mathrm{CN}, 70{ }^{\circ} \mathrm{C}\right) \delta 8.46(\mathrm{br} \mathrm{s}, 1 \mathrm{H}), 8.18(\mathrm{~s}, 1 \mathrm{H}), 8.05(\mathrm{~s}, 1 \mathrm{H}), 8.02(\mathrm{br} \mathrm{s}, 1$ H), 8.01 (s, 1 H), $7.97-7.91$ (m, 1 H), 7.75 (br s, 1 H), 7.67 (br s, 1 H), 7.60 - 7.56 (m, 2 H), $7.54(\mathrm{~s}, 1 \mathrm{H}), 7.51-7.47$ (m, $1 \mathrm{H}), 7.31-7.27$ (m, $4 \mathrm{H}), 7.26-7.21$ (m, $4 \mathrm{H}), 7.19$ (d, $J=8.1$ Hz, $1 \mathrm{H}), 6.90(\mathrm{~d}, J=10.0 \mathrm{~Hz}, 1 \mathrm{H}), 6.44(\mathrm{~s}, 1 \mathrm{H}), 6.37$ (dd, $J=8.9,4.7 \mathrm{~Hz}, 1 \mathrm{H}), 6.02-5.97$ (m, $1 \mathrm{H}), 5.93-5.90(\mathrm{br}, 1 \mathrm{H}), 5.85(\mathrm{~d}, J=1.0 \mathrm{~Hz}, 1 \mathrm{H}), 5.81-5.74(\mathrm{~m}, 1 \mathrm{H}), 5.70-5.65$ (br, 2 H), $5.44(\mathrm{~d}, J=8.8 \mathrm{~Hz}, 1 \mathrm{H}), 5.37$ (dq, $J=17.2,1.5 \mathrm{~Hz}, 1 \mathrm{H}), 5.26$ (dq, $J=10.7,1.5 \mathrm{~Hz}, 1 \mathrm{H}$ ), $5.22-5.18(\mathrm{~m}, 2 \mathrm{H}), 5.07(\mathrm{~d}, J=10.1 \mathrm{~Hz}, 1 \mathrm{H}), 4.91-4.84(\mathrm{~m}, 2 \mathrm{H}), 4.74-4.68(\mathrm{~m}, 3 \mathrm{H}), 4.63$ - 4.59 (m, 1 H), 4.51 - 4.42 (m, 3 H), 4.31 - 4.24 (m, 3 H), 4.06 - 4.02 (m, 2 H), 3.64 - 3.58 (m, $2 \mathrm{H}), 3.46-3.37$ (m, $4 \mathrm{H}), 3.33$ (dd, $J=12.9,5.5 \mathrm{~Hz}, 1 \mathrm{H}), 3.21$ (dd, $J=12.9,7.3 \mathrm{~Hz}, 1 \mathrm{H}), 3.21$ $-3.18(\mathrm{~m}, 1 \mathrm{H}), 3.10$ (d, $J=5.9 \mathrm{~Hz}, 1 \mathrm{H}), 2.99-2.92$ (br, $1 \mathrm{H}), 2.58-2.51$ (br, $1 \mathrm{H}), 1.36$ - 
$1.34(\mathrm{~m}, 12 \mathrm{H}), 1.34-1.29(\mathrm{~m}, 9 \mathrm{H}), 1.13(\mathrm{~d}, J=6.2 \mathrm{~Hz}, 3 \mathrm{H}), 0.99-0.89(\mathrm{~m}, 36 \mathrm{H}), 0.82(\mathrm{~d}, J$ $=7.0 \mathrm{~Hz}, 3 \mathrm{H}), 0.74(\mathrm{~s}, 9 \mathrm{H}), 0.65(\mathrm{q}, J=7.9 \mathrm{~Hz}, 6 \mathrm{H}), 0.21(\mathrm{~s}, 3 \mathrm{H}), 0.10(\mathrm{~s}, 3 \mathrm{H}), 0.09(\mathrm{~s}, 3 \mathrm{H})$, 0.09 (s, $3 \mathrm{H}), 0.07$ (s, $3 \mathrm{H}), 0.08$ (s, $3 \mathrm{H}),-0.04$ (s, $3 \mathrm{H}),-0.16$ (s, $3 \mathrm{H}) ;{ }^{13} \mathrm{C}$ NMR $(125 \mathrm{MHz}$, dioxane- $\left.d_{8}, 70{ }^{\circ} \mathrm{C}\right) \delta 174.5,173.7,173.4,172.2,171.9,170.7,170.0,169.6,164.0,161.2,160.4$, $158.9,157.5,151.3,133.3,133.1,131.1,133.0,132.7,129.8,129.7,127.7,127.5,118.6,117.9$, $75.4,71.6,71.2,64.9,62.5,57.9,54.0,53.7,52.4,49.7,46.8,39.3,34.0,32.5,30.2,30.1,29.8$, 29.6, 29.5, 27.7, 26.5, 26.5, 26.2, 26.0, 25.5, 23.1, 18.9, 18.7, 18.6, 18.5, 18.4, 16.1, 14.2, 11.6, 7.1, 5.6, -3.2, -3.9, -4.6, -4.7; HRMS (ESI-TOF) calcd for $\mathrm{C}_{121} \mathrm{H}_{179} \mathrm{~N}_{19} \mathrm{O}_{22} \mathrm{~S}_{5} \mathrm{Se}_{2} \mathrm{Si}_{5} \mathrm{Na}^{+}[\mathrm{M}+$ $\left.\mathrm{Na}^{+}\right]$2732.9144, found 2732.9132 .

\section{Triene seco compound 16 (Scheme 2):}

To bis-phenylselenium seco compound 15 (12.5 mg, $0.0046 \mathrm{mmol})$ and $\mathrm{NaHCO}_{3}(12 \mathrm{mg}, 0.14$ mmol) at $0{ }^{\circ} \mathrm{C}$ was added $1.0 \mathrm{~mL}$ of a 1:10 mixture of $t$-BuOOH (5-6 M in decane): $\mathrm{CH}_{2} \mathrm{Cl}_{2}$.

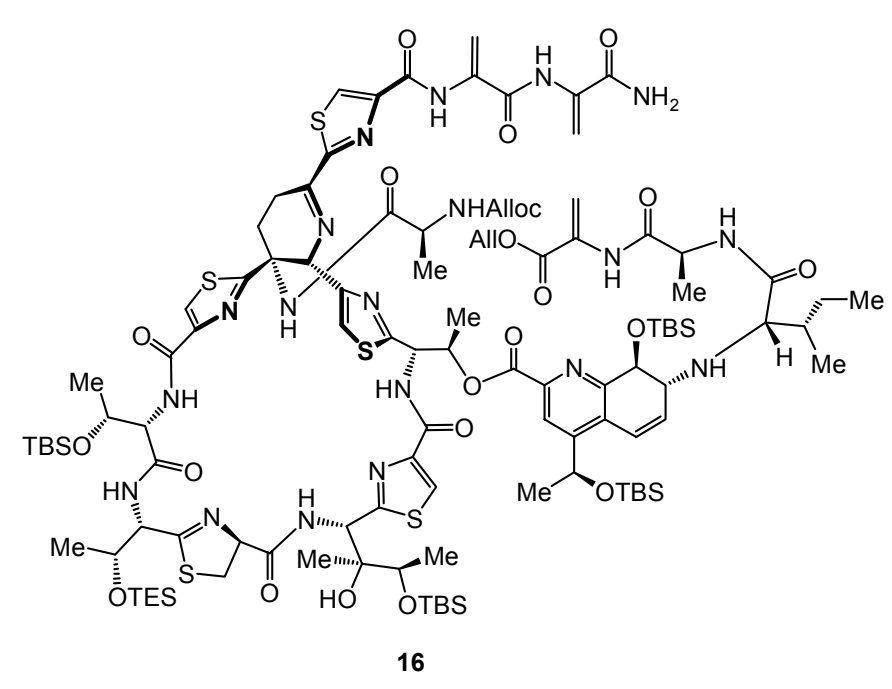

The reaction mixture was stirred for 5 min at $0{ }^{\circ} \mathrm{C}$ and $30 \mathrm{~min}$ at $25{ }^{\circ} \mathrm{C}$. A further $2.0 \mathrm{~mL}$ of the $\mathrm{CH}_{2} \mathrm{Cl}_{2}: t-\mathrm{BuOOH}$ solution was added and stirring was continued for $45 \mathrm{~min}$. The reaction mixture was cooled to $0{ }^{\circ} \mathrm{C}$ and quenched with a saturated aqueous 1:1 solution of $\mathrm{NaHCO}_{3}$ and $\mathrm{Na}_{2} \mathrm{~S}_{2} \mathrm{O}_{3}(6.0 \mathrm{~mL})$. The aqueous layer was extracted with $\mathrm{CH}_{2} \mathrm{Cl}_{2}$ $(3 \times 5 \mathrm{~mL})$ and the combined organic

layers were dried $\left(\mathrm{Na}_{2} \mathrm{SO}_{4}\right)$ and concentrated. The residue was purified by preparative TLC (silica gel, $\mathrm{CH}_{2} \mathrm{Cl}_{2}: \mathrm{MeOH}, 92: 8$ ) to give triene seco compound 16 as a yellow oil (7.5 mg, 68\%). 16: $R_{\mathrm{f}}=0.34$ (silica gel, $\left.\mathrm{CH}_{2} \mathrm{Cl}_{2}: \mathrm{MeOH}, 93: 7\right) ;[\alpha]_{\mathrm{D}}{ }^{32}=-33.0\left(\mathrm{CHCl}_{3}, c=1.00\right)$; IR (film) $v_{\max }$ 3362, 2922, 2852, 1701, 1655, 1522, 1459, 1255, 1101, 836, $756 \mathrm{~cm}^{-1}$; ${ }^{1} \mathrm{H}$ NMR $(600 \mathrm{MHz}$, $\left.\mathrm{CD}_{3} \mathrm{CN}, 70{ }^{\circ} \mathrm{C}\right) \delta 9.76($ br s, $1 \mathrm{H}), 8.89($ br s, $1 \mathrm{H}), 8.45$ (br s, $\left.1 \mathrm{H}\right), 8.27(\mathrm{~s}, 1 \mathrm{H}), 8.05(\mathrm{~s}, 1 \mathrm{H})$, $8.01(\mathrm{~s}, 1 \mathrm{H}), 7.97-7.93(\mathrm{~m}, 1 \mathrm{H}), 7.90(\mathrm{~d}, J=6.2 \mathrm{~Hz}, 1 \mathrm{H}), 7.75$ (br s, $1 \mathrm{H}), 7.68$ (br s, $1 \mathrm{H})$, 7.54 (s, $1 \mathrm{H}), 7.30$ (d, $J=6.6 \mathrm{~Hz}, 1 \mathrm{H}), 6.90(\mathrm{~d}, J=10.0 \mathrm{~Hz}, 1 \mathrm{H}), 6.52(\mathrm{~d}, J=0.8 \mathrm{~Hz}, 1 \mathrm{H}), 6.44$ (s, $1 \mathrm{H}), 6.39-6.34(\mathrm{~m}, 1 \mathrm{H}), 6.38(\mathrm{~s}, 1 \mathrm{H}), 6.00-5.96(\mathrm{~m}, 1 \mathrm{H}), 5.94-5.91(\mathrm{br}, 1 \mathrm{H}), 5.85(\mathrm{~s}$, 
$1 \mathrm{H}), 5.79-5.73(\mathrm{~m}, 1 \mathrm{H}), 5.69-5.65(\mathrm{br}, 2 \mathrm{H}), 5.58(\mathrm{~s}, 1 \mathrm{H}), 5.56(\mathrm{~s}, 1 \mathrm{H}), 5.44(\mathrm{~d}, J=8.3 \mathrm{~Hz}$, $1 \mathrm{H}), 5.36(\mathrm{~d}, J=17.5 \mathrm{~Hz}, 1 \mathrm{H}), 5.26(\mathrm{~d}, J=10.6 \mathrm{~Hz}, 1 \mathrm{H}), 5.21-5.14(\mathrm{~m}, 2 \mathrm{H}), 5.07(\mathrm{~d}, J=$ $11.0 \mathrm{~Hz}, 1 \mathrm{H}), 4.91-4.84$ (m, $2 \mathrm{H}), 4.73-4.68$ (m, $3 \mathrm{H}), 4.63-4.59$ (m, $1 \mathrm{H}), 4.48-4.39$ (m, 3 H), $4.29-4.21$ (m, $3 \mathrm{H}), 4.08-4.01(\mathrm{~m}, 2 \mathrm{H}), 3.66-3.58(\mathrm{~m}, 3 \mathrm{H}), 3.46-3.39(\mathrm{~m}, 2 \mathrm{H}), 3.27$ (dd, $J=12.9,5.5 \mathrm{~Hz}, 1 \mathrm{H}), 3.09$ (d, $J=5.3 \mathrm{~Hz}, 1 \mathrm{H}), 3.01-2.95$ (m, $1 \mathrm{H}), 2.58-2.51$ (br, $1 \mathrm{H})$, $1.38-1.34$ (m, 9 H), $1.34-1.28$ (m, 9 H), 1.25 (d, $J=6.1 \mathrm{~Hz}, 3 \mathrm{H}), 1.13$ (d, $J=6.2 \mathrm{~Hz}, 3 \mathrm{H})$, 0.98 (s, 9 H), 0.96 (t, $J=7.9 \mathrm{~Hz}, 9$ H), 0.91 (s, 9 H), 0.88 (s, 9 H), 0.76 (d, $J=5.7 \mathrm{~Hz}, 3 \mathrm{H}), 0.73$ (s, 9 H), 0.64 (q, $J=7.9$ Hz, 6 H), 0.21 (s, 6 H), 0.09 (s, 6 H), 0.08 (s, 3 H), 0.07 (s, 3 H), -0.04 $(\mathrm{s}, 3 \mathrm{H}),-0.16(\mathrm{~s}, 3 \mathrm{H}) ;{ }^{13} \mathrm{C}$ NMR $\left(125 \mathrm{MHz}\right.$, dioxane- $\left.d_{8}, 70{ }^{\circ} \mathrm{C}\right) \delta 173.9,171.3,169.0,163.3$, 162.4, 159.8, 159.1, 151.2, 135.3, 134.4, 132.1, 129.9, 129.8, 118.0, 117.1, 111.4, 102.0, 79.8, 74.8, 73.0, 71.0, 70.6, 70.5, 68.2, 64.3, 61.9, 61.4, 57.9, 54.0, 52.4, 49.1, 46.2, 38.7, 34.1, 33.4, $31.9,29.6,29.5,29.4,29.2,27.1,25.9,25.8,25.6,25.4,24.9,23.2,19.4,18.3,17.8,17.7,15.5$, 13.5, 11.0, 6.5, 5.0, -4.6, -5.0, -5.2, -5.3; HRMS (ESI-TOF) calcd for $\mathrm{C}_{109} \mathrm{H}_{167} \mathrm{~N}_{19} \mathrm{O}_{22} \mathrm{~S}_{5} \mathrm{Si}_{5} \mathrm{Na}^{+}$ $\left[\mathrm{M}+\mathrm{Na}^{+}\right] 2416.9875$, found 2416.9912 .

\section{Bis-dehydroalanine thiazoline macrocycle 18 (Scheme 3):}

To a suspension of bis-phenylselenium macrocycle $13(20 \mathrm{mg}, 0.010 \mathrm{mmol})$ and $\mathrm{NaHCO}_{3}(20$ $\mathrm{mg}, 0.24 \mathrm{mmol})$ in $\mathrm{CH}_{2} \mathrm{Cl}_{2}(0.5 \mathrm{~mL})$ at $0{ }^{\circ} \mathrm{C}$ was added $t-\mathrm{BuOOH}(0.10 \mathrm{~mL}$ of a $5-6 \mathrm{M}$ solution

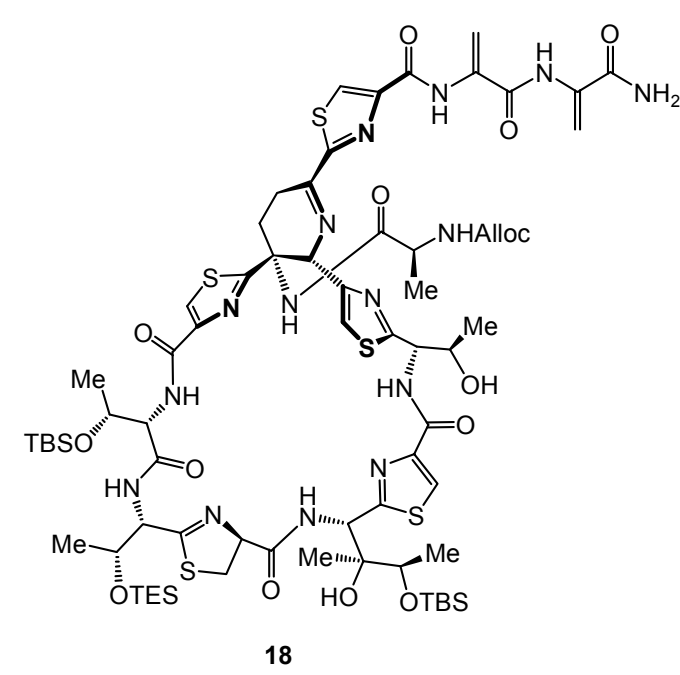
in decane). The reaction mixture was stirred for 5 min at $0{ }^{\circ} \mathrm{C}$ and then warmed to $25^{\circ} \mathrm{C}$. Stirring was continued for $1 \mathrm{~h}$ at $25^{\circ} \mathrm{C}$, then a further $0.10 \mathrm{~mL}$ of the $5-6 \mathrm{M}$ decane solution of $t-\mathrm{BuOOH}$ was added. Stirring was continued for $1 \mathrm{~h}$, then the reaction mixture was cooled to $0{ }^{\circ} \mathrm{C}$ and EtOAc $(12 \mathrm{~mL})$ and a saturated aqueous 1:1 solution of $\mathrm{Na}_{2} \mathrm{~S}_{2} \mathrm{O}_{3}$ and $\mathrm{NaHCO}_{3}(7 \mathrm{~mL})$ was added. The layers were separated and the aqueous phase was re-extracted with EtOAc $(2 \times 5 \mathrm{~mL})$. The combined organic

layers were dried $\left(\mathrm{Na}_{2} \mathrm{SO}_{4}\right)$ and concentrated. The residue was purified by preparative TLC (silica gel, $\left.\mathrm{CH}_{2} \mathrm{Cl}_{2}: \mathrm{MeOH}, 93: 7\right)$ to give bis-dehydroalanine thiazoline macrocycle 18 (14.1 mg, $84 \%)$ as a yellow oil. 
18: $R_{\mathrm{f}}=0.35$ (silica gel, $\left.\mathrm{CH}_{2} \mathrm{Cl}_{2}: \mathrm{MeOH}, 93: 7\right) ;[\alpha]_{\mathrm{D}}{ }^{32}=+15.0\left(\mathrm{CHCl}_{3}, c=1.00\right)$; IR (film) $v_{\max }$ $3367,2927,2855,1683,1528,1484,1382,1256,1101,833 \mathrm{~cm}^{-1} ;{ }^{1} \mathrm{H}$ NMR $\left(500 \mathrm{MHz}, \mathrm{CD}_{3} \mathrm{CN}\right.$, $\left.70{ }^{\circ} \mathrm{C}\right) \delta 9.77$ (br s, $\left.1 \mathrm{H}\right), 8.90$ (br s, $\left.1 \mathrm{H}\right), 8.48$ - 8.42 (br, $\left.1 \mathrm{H}\right), 8.26$ (s, $\left.1 \mathrm{H}\right), 8.16$ (s, $\left.1 \mathrm{H}\right), 7.89$ $(\mathrm{d}, J=8.8 \mathrm{~Hz}, 1 \mathrm{H}), 7.80$ (d, $J=7.9 \mathrm{~Hz}, 1 \mathrm{H}), 7.60-7.52(\mathrm{br}, 1 \mathrm{H}), 7.39$ (s, $1 \mathrm{H}), 6.52$ (d, $J=1.9$ $\mathrm{Hz}, 1 \mathrm{H}), 6.38$ (d, J=1.5 Hz, $1 \mathrm{H}), 5.97-5.91$ (br, $1 \mathrm{H}), 5.79-5.72(\mathrm{~m}, 1 \mathrm{H}), 5.58$ (s, $1 \mathrm{H}), 5.56$ (t, $J=1.8 \mathrm{~Hz}, 1 \mathrm{H}), 5.39-5.32(\mathrm{~m}, 1 \mathrm{H}), 5.27-5.23(\mathrm{br}, 1 \mathrm{H}), 5.17(\mathrm{dq}, J=16.8,1.9 \mathrm{~Hz}, 1 \mathrm{H})$, 5.07 (dq, $J=10.3,1.1 \mathrm{~Hz}, 1 \mathrm{H}), 4.98$ (dt, $J=9.2,1.4 \mathrm{~Hz}, 1 \mathrm{H}), 4.86-4.84$ (m, $1 \mathrm{H}), 4.72-4.68$ (br, $1 \mathrm{H}), 4.62-4.59$ (br, $1 \mathrm{H}), 4.29-4.26$ (m, $4 \mathrm{H}), 4.11$ (q, $J=6.3 \mathrm{~Hz}, 1 \mathrm{H}), 3.97$ (quin, $J=7.0$ $\mathrm{Hz}, 1 \mathrm{H}), 3.68(\mathrm{dd}, J=13.2,6.3 \mathrm{~Hz}, 1 \mathrm{H}), 3.58-3.54(\mathrm{~m}, 5 \mathrm{H}), 3.31-3.24$ (m, $1 \mathrm{H}), 2.97-2.89$ $(\mathrm{m}, 1 \mathrm{H}), 2.47(\mathrm{dt}, J=12.5,7.0 \mathrm{~Hz}, 1 \mathrm{H}), 2.26(\mathrm{~m}, 1 \mathrm{H}), 1.32(\mathrm{~d}, J=6.1 \mathrm{~Hz}, 3 \mathrm{H}), 1.25(\mathrm{~d}, J=$ $6.6 \mathrm{~Hz}, 6 \mathrm{H}), 1.21(\mathrm{~d}, J=6.6 \mathrm{~Hz}, 3 \mathrm{H}), 1.10$ (d, $J=6.3 \mathrm{~Hz}, 3 \mathrm{H}), 1.04$ (s, $3 \mathrm{H}), 0.98(\mathrm{~s}, 9 \mathrm{H}), 0.95$ (s, 9 H), 0.94 (t, $J=7.7 \mathrm{~Hz}, 9 \mathrm{H}), 0.64$ (q, $J=7.7 \mathrm{~Hz}, 6 \mathrm{H}), 0.20$ (s, $3 \mathrm{H}), 0.19$ (s, $3 \mathrm{H}), 0.12$ (s, 3 $\mathrm{H}), 0.089(\mathrm{~s}, 3 \mathrm{H}) ;{ }^{13} \mathrm{C}$ NMR $\left(125 \mathrm{MHz}\right.$, dioxane- $\left.d_{8}, 70{ }^{\circ} \mathrm{C}\right) \delta 174.5,173.6,173.1,170.4,169.7$, 169.3, 166.2, 163.0, 159.6, 156.5, 151.6, 149.6, 135.8, 135.0, 134.0, 128.0, 119.5, 117.8, 102.6, 71.1, 52.1, 34.0, 32.5, 30.2, 30.1, 30.0, 29.9, 29.8, 26.5, 26.4, 25.5, 25.1, 23.1, 18.5, 17.9, 14.2, 7.0, 5.5, -3.3, -4.9; HRMS (ESI-TOF) calcd for $\mathrm{C}_{70} \mathrm{H}_{106} \mathrm{~N}_{15} \mathrm{O}_{15} \mathrm{~S}_{5} \mathrm{Si}_{3}{ }^{+}\left[\mathrm{M}+\mathrm{H}^{+}\right] 1640.5898$, found 1640.5892 .

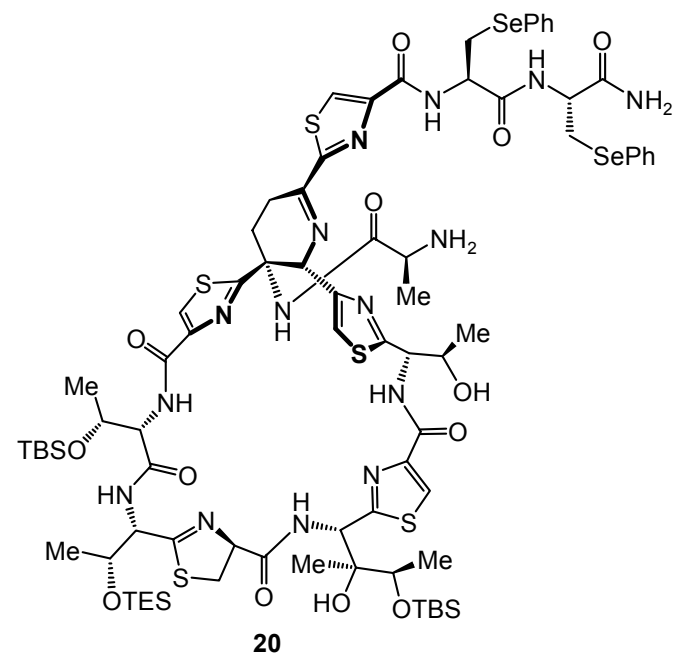

Bis-phenylselenium thiazoline macrocycle amine 20 (Scheme 3):

See below (preparation of seco ester 32) for the deprotection conditions of bis-phenylselenium thiazoline macrocycle $\mathbf{1 3}$ to free amine $\mathbf{2 0 .}$ 


\section{Bis-phenylselenium model system 23 (Scheme 4):}

To thiazole $21^{5}(1.0 \mathrm{~g}, 2.35 \mathrm{mmol})$ in 1,2-dichloroethane $(12.0 \mathrm{~mL})$ at $25{ }^{\circ} \mathrm{C}$ was added trimethyltin hydroxide $(1.30 \mathrm{~g}, 7.05 \mathrm{mmol})$. The reaction mixture was refluxed for $1 \mathrm{~h}$, then a

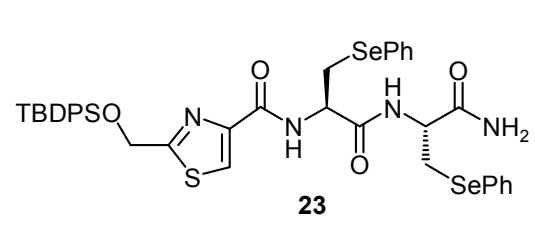

further $1.30 \mathrm{~g}$ of trimethyltin hydroxide $(7.05 \mathrm{mmol})$ was added and reflux was continued for a further hour. The reaction mixture was cooled to $25{ }^{\circ} \mathrm{C}$ and concentrated, and the residue was taken up in EtOAc $(30 \mathrm{~mL})$. The organic layer was washed with aqueous $5 \% \mathrm{HCl}$ solution $(5 \times 30 \mathrm{~mL})$ and brine $(30 \mathrm{~mL})$, then dried $\left(\mathrm{Na}_{2} \mathrm{SO}_{4}\right)$ and concentrated to give pure carboxylic acid 22 as a white foam (932 $\mathrm{mg}, 100 \%)$. To carboxylic acid $22(100 \mathrm{mg}, 0.24 \mathrm{mmol})$ and amine $4^{1}(114 \mathrm{mg}, 0.24 \mathrm{mmol})$ in DMF $(1.2 \mathrm{~mL})$ at $0{ }^{\circ} \mathrm{C}$ were added $i$-Pr 2 NEt $(0.13 \mathrm{~mL}, 0.72 \mathrm{mmol})$, HOAt $(49 \mathrm{mg}, 0.36 \mathrm{mmol})$, and HATU (137 $\mathrm{mg}, 0.36 \mathrm{mmol}$ ). The reaction mixture was stirred for $20 \mathrm{~min}$ at $0{ }^{\circ} \mathrm{C}$ and $2 \mathrm{~h}$ at $25{ }^{\circ} \mathrm{C}$, then diluted with EtOAc $(40 \mathrm{~mL})$. The organic layer was washed with aqueous $5 \% \mathrm{HCl}$ solution, water, saturated aqueous $\mathrm{NaHCO}_{3}$ solution, and brine $\left(20 \mathrm{~mL}\right.$ each), then dried $\left(\mathrm{Na}_{2} \mathrm{SO}_{4}\right)$ and concentrated. The residue was purified by flash column chromatography (silica gel, hexanes:EtOAc, 2:8) to give bis-phenylselenium model system $23(181 \mathrm{mg}, 85 \%)$ as a clear oil. 23: $R_{\mathrm{f}}=0.49$ (silica gel, hexanes:EtOAc, 3:7); $[\alpha]_{\mathrm{D}}{ }^{32}=-82.4\left(\mathrm{CHCl}_{3}, c=1.00\right)$; IR (film) $v_{\max }$ 3365, 2931, 2849, 1654, 1559, 1474, 1111, 738, $702 \mathrm{~cm}^{-1} ;{ }^{1} \mathrm{H}$ NMR $\left(500 \mathrm{MHz}, \mathrm{CDCl}_{3}\right) \delta 8.09$ (s, $1 \mathrm{H}), 7.77(\mathrm{~d}, J=6.3 \mathrm{~Hz}, 1 \mathrm{H}) 7.74-7.70(\mathrm{~m}, 4 \mathrm{H}), 7.49-7.41(\mathrm{~m}, 10 \mathrm{H}), 7.19-7.14(\mathrm{~m} 6$ H), 6.94 (d, $J=8.1 \mathrm{~Hz}, 1 \mathrm{H}), 6.65$ (br s, $1 \mathrm{H}), 5.41$ (br s, $1 \mathrm{H}$ ), 4.94 (s, $2 \mathrm{H}), 4.65$ (dt, $J=8.1,5.9$ $\mathrm{Hz}, 1 \mathrm{H}), 4.52(\mathrm{q}, J=6.3 \mathrm{~Hz}, 1 \mathrm{H}), 3.43(\mathrm{dd}, J=13.2,5.5 \mathrm{~Hz}, 1 \mathrm{H}), 3.26(\mathrm{dd}, J=12.9,5.9 \mathrm{~Hz}, 1$ H), $3.15(\mathrm{dd}, J=13.2,7.0 \mathrm{~Hz}, 1 \mathrm{H}), 3.12(\mathrm{dd}, J=13.2,5.9 \mathrm{~Hz}), 1.16(\mathrm{~s}, 9 \mathrm{H}) ;{ }^{13} \mathrm{C}$ NMR $(150$ $\left.\mathrm{MHz}, \mathrm{CDCl}_{3}\right) \delta 173.3,172.3,170.1,161.8,148.8,135.7,135.6,133.3,133.2,132.4,132.3$, 130.4, 129.4, 129.3, 128.6, 128.2, 127.7, 127.6, 124.2, 63.8, 53.8, 53.0, 38.8, 29.1, 29.0, 26.9, 19.4, 14.5; HRMS (ESI-TOF) calcd for $\mathrm{C}_{39} \mathrm{H}_{43} \mathrm{~N}_{4} \mathrm{O}_{4} \mathrm{SSe}_{2}{ }^{+}\left[\mathrm{M}+\mathrm{H}^{+}\right]$851.1099, found 851.1085.

\section{Bis-dehydroalanine model system 24 (Scheme 4):}

To bis-phenylselenium model system $23(90 \mathrm{mg}, 0.11 \mathrm{mmol})$ in $\mathrm{CH}_{2} \mathrm{Cl}_{2}(1.0 \mathrm{~mL})$ at $0{ }^{\circ} \mathrm{C}$ was added $t$ - $\mathrm{BuOOH}(0.20 \mathrm{~mL}$ of a $5-6 \mathrm{M}$ solution in decane). The reaction mixture was stirred for $10 \mathrm{~min}$ at $0{ }^{\circ} \mathrm{C}$ and $30 \mathrm{~min}$ at $25^{\circ} \mathrm{C}$, then a further $0.20 \mathrm{~mL}$ of the $t$-BuOOH solution was added. Stirring was continued for $30 \mathrm{~min}$, then a third $0.20 \mathrm{~mL}$ portion of the $t-\mathrm{BuOOH}$ solution was 
added and stirring was continued for $30 \mathrm{~min}$. The reaction mixture was then cooled to $0{ }^{\circ} \mathrm{C}$ and quenched with a saturated aqueous $1: 1$ solution of $\mathrm{NaHCO}_{3}$ and $\mathrm{Na}_{2} \mathrm{~S}_{2} \mathrm{O}_{3}(5 \mathrm{~mL})$. The aqueous layer was extracted with $\mathrm{CH}_{2} \mathrm{Cl}_{2}(3 \times 10 \mathrm{~mL})$, and the

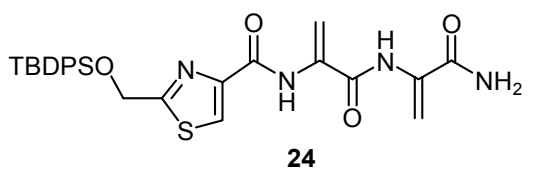
combined organic layers were washed with brine $(10 \mathrm{~mL})$, dried $\left(\mathrm{Na}_{2} \mathrm{SO}_{4}\right)$, and concentrated. The residue was purified by flash column chromatography (silica gel, hexanes:EtOAc, 4:6 to give bis-dehydroalanine model system 24 (46 $\mathrm{mg}, 82 \%)$ as a white powder.

24: $R_{\mathrm{f}}=0.61$ (silica gel, hexanes:EtOAc, 3:7); IR (film) $v_{\max } 3366,2931,2847,1658,1486$, 1427, 1192, 1111, 891, 823, 743, $701 \mathrm{~cm}^{-1} ;{ }^{1} \mathrm{H}$ NMR (500 MHz, $\left.\mathrm{CDCl}_{3}\right) \delta 9.81$ (br s, $\left.1 \mathrm{H}\right), 8.93$ (br s, $1 \mathrm{H}), 8.14(\mathrm{~s}, 1 \mathrm{H}), 7.71-7.68(\mathrm{~m}, 4 \mathrm{H}), 7.49-7.40(\mathrm{~m}, 6 \mathrm{H}), 6.73(\mathrm{~d}, J=2.2 \mathrm{~Hz}, 1 \mathrm{H})$, $6.61(\mathrm{~d}, J=2.2 \mathrm{~Hz}, 1 \mathrm{H}), 6.15(\mathrm{br}, 1 \mathrm{H}), 5.78(\mathrm{br}, 1 \mathrm{H}), 5.50(\mathrm{t}, J=1.9 \mathrm{~Hz}, 1 \mathrm{H}), 5.38(\mathrm{t}, J=1.5$ $\mathrm{Hz}, 1 \mathrm{H}), 4.98$ (s, $2 \mathrm{H}), 1.15$ (s, $9 \mathrm{H}) ;{ }^{13} \mathrm{C} \mathrm{NMR}\left(125 \mathrm{MHz}, \mathrm{CDCl}_{3}\right) \delta 173.3,165.6,162.4,160.1$, $149.9,135.7,134.6,133.1,132.5,130.4,128.2,123.9,103.4,102.9,64.0,26.9,19.5$; HRMS (ESI-TOF) calcd for $\mathrm{C}_{27} \mathrm{H}_{31} \mathrm{~N}_{4} \mathrm{O}_{4} \mathrm{SSi}^{+}\left[\mathrm{M}+\mathrm{H}^{+}\right]$535.1830, found 535.1829.

\section{Reduced dehydroalanines 25 and $25^{\prime}$ (14:1 mixture of inseparable regioisomers) [Scheme 5]:}

To bis-dehydroalanine model system $24(22 \mathrm{mg}, 0.041 \mathrm{mmol})$ in $\mathrm{THF}(0.40 \mathrm{~mL})$ at $0{ }^{\circ} \mathrm{C}$ was added $n-\mathrm{Bu}_{3} \mathrm{SnH}(0.28 \mathrm{~mL}, 1.03 \mathrm{mmol})$ and $\mathrm{PdCl}_{2}\left(\mathrm{PPh}_{3}\right)_{2}(2.9 \mathrm{mg}, 0.0041 \mathrm{mmol})$. The reaction

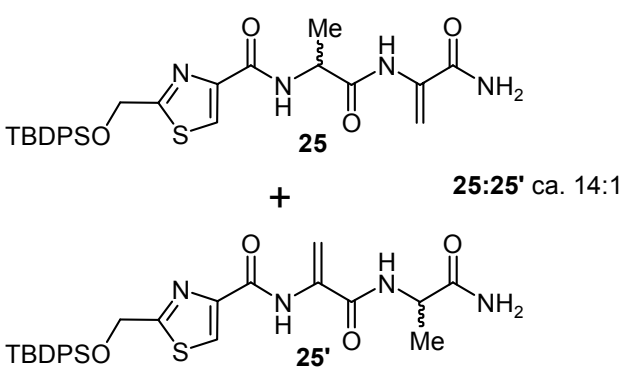
mixture was stirred for $10 \mathrm{~min}$ at $0{ }^{\circ} \mathrm{C}$ and then for $1 \mathrm{~h}$ at $25{ }^{\circ} \mathrm{C}$. A further $2.9 \mathrm{mg}$ of $\mathrm{PdCl}_{2}\left(\mathrm{PPh}_{3}\right)_{2}(0.0041$ $\mathrm{mmol})$ and $0.28 \mathrm{~mL}$ of $n-\mathrm{Bu}_{3} \mathrm{SnH}(1.03 \mathrm{mmol})$ were then added and stirring was continued for $50 \mathrm{~min}$. The crude reaction mixture was then purified by flash column chromatography (silica gel, hexanes:EtOAc, 4:6) to give reduced dehydroalanines $\mathbf{2 5}$ and $\mathbf{2 5}^{\prime}(18.9 \mathrm{mg}, 85 \%)$ as a yellow oil and as a $\mathrm{ca} 14: 1$ mixture of chromatographically inseparable regioisomers.

25 and 25' ( ca 14:1 mixture of chromatographically inseparable regioisomers): $R_{\mathrm{f}}=0.48$ (silica gel, hexanes:EtOAc, 3:7); IR (film) $v_{\max }$ 3355, 2930, 2857, 1654, 1522, 1427, 1193, 1113, 823, 754, $701 \mathrm{~cm}^{-1} ;{ }^{1} \mathrm{H}$ NMR (600 MHz, $\mathrm{CDCl}_{3}$ ), major regioisomer $25 \delta 8.76(\mathrm{br} \mathrm{s}, 1 \mathrm{H}), 8.07(\mathrm{~s}, 1$ H), $7.68-7.62(\mathrm{~m}, 4 \mathrm{H}), 7.47-7.37$ (m, $6 \mathrm{H}), 6.48$ (d, J=1.7 Hz, $1 \mathrm{H}), 6.19$ (br, $1 \mathrm{H}), 5.91$ (br, 
$1 \mathrm{H}), 5.33$ (br s , $1 \mathrm{H}$ ), 4.91 (s, $2 \mathrm{H}$ ), 4.82 (quin, $J=7.4 \mathrm{~Hz}, 1 \mathrm{H}$ ), 1.47 (d, $J=7.0 \mathrm{~Hz}, 3 \mathrm{H}$ ), 1.11 (s, 9 H), minor regioisomer 25' $\delta 9.72(\mathrm{br} \mathrm{s}, 1 \mathrm{H}), 8.09$ (s, $1 \mathrm{H}), 7.68-7.62(\mathrm{~m}, 4 \mathrm{H}), 7.47-7.37$ (m, $6 \mathrm{H}), 7.00$ (br d, $J=7.0 \mathrm{~Hz}, 1 \mathrm{H}) 6.53$ (d, $J=1.7 \mathrm{~Hz}, 1 \mathrm{H}), 6.19$ (br, $1 \mathrm{H}), 5.91$ (br, $1 \mathrm{H}$ ), 5.41 (br s , 1 H), 4.94 (s, 2 H), 4.53 (quin, $J=7.0 \mathrm{~Hz}, 1 \mathrm{H}$ ), 1.43 (d, $J=7.0 \mathrm{~Hz}, 3 \mathrm{H}$ ), 1.11 (s, 9 $\mathrm{H}) ;{ }^{13} \mathrm{C}$ NMR $\left(150 \mathrm{MHz}, \mathrm{CDCl}_{3}\right) \delta$ 173.2, 171.3, 165.9, 161.4, 149.3, 135.7, 133.7, 132.5, 132.4, 132.3, 132.2, 130.3, 128.8, 128.7, 128.2, 103.9, 63.8, 49.8, 26.9, 19.5, 18.4; HRMS (ESI-TOF) calcd for $\mathrm{C}_{27} \mathrm{H}_{33} \mathrm{~N}_{4} \mathrm{O}_{4} \mathrm{SSi}^{+}\left[\mathrm{M}+\mathrm{H}^{+}\right]$537.1986, found 537.1982.

\section{Seco ester 32 (Scheme 7):}

To bis-phenylselenium thiazoline macrocycle $13(51 \mathrm{mg}, 0.026 \mathrm{mmol})$ in $\mathrm{CH}_{2} \mathrm{Cl}_{2}(0.51 \mathrm{~mL})$ at $45^{\circ} \mathrm{C}$ was added $n$ - $\mathrm{Bu}_{3} \mathrm{SnH}(0.35 \mathrm{~mL}, 1.30 \mathrm{mmol})$ and $\mathrm{PdCl}_{2}\left(\mathrm{PPh}_{3}\right)_{2}(1.8 \mathrm{mg}, 0.0026 \mathrm{mmol}$ in 50

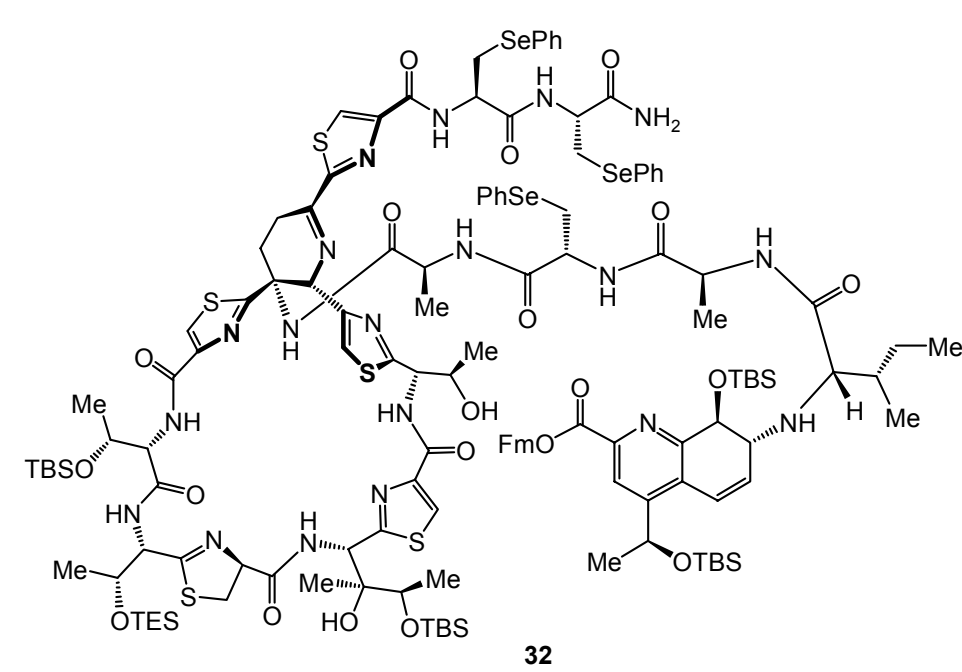

$\mu \mathrm{L}$ of $\mathrm{CH}_{2} \mathrm{Cl}_{2}$ ). The reaction mixture was stirred for $5 \mathrm{~min}$ at $-45^{\circ} \mathrm{C}$ and then warmed to $0{ }^{\circ} \mathrm{C}$ and stirred for a further $30 \mathrm{~min}$. The crude reaction mixture was then directly purified by preparative TLC (silica gel, hexanes, then $\left.\mathrm{CH}_{2} \mathrm{Cl}_{2}: \mathrm{MeOH}, 92.5: 7.5\right)$ to give thiazoline macrocycle amine $\mathbf{2 0}$ (42 $\mathrm{mg}, 86 \%$ ) as a yellow foam. To quinaldic acid $\mathbf{3 1}^{1}(27 \mathrm{mg}, 0.025$

$\mathrm{mmol})$ and thiazoline macrocycle amine $20(42 \mathrm{mg}, 0.022 \mathrm{mmol})$ in DMF $(0.50 \mathrm{~mL})$ at $0{ }^{\circ} \mathrm{C}$ was added HOAt $(3.7 \mathrm{mg}, 0.027 \mathrm{mmol})$, followed by HATU $(10.2 \mathrm{mg}, 0.027 \mathrm{mmol})$. The reaction mixture was stirred for $15 \mathrm{~min}$ at $0{ }^{\circ} \mathrm{C}$ then $i-\operatorname{Pr}_{2} \mathrm{NEt}(12 \mu \mathrm{L}, 0.070 \mathrm{mmol})$ was added and stirring was continued for a further $45 \mathrm{~min}$. The resulting mixture was then diluted with EtOAc $(5 \mathrm{~mL})$ and the organic layer was washed with water and brine (1.5 mL each), dried $\left(\mathrm{Na}_{2} \mathrm{SO}_{4}\right)$ and concentrated. The residue was purified by preparative TLC (silica gel, $\mathrm{CH}_{2} \mathrm{Cl}_{2}: \mathrm{MeOH}, 93: 7$ ) to give seco ester 32 (44 $\mathrm{mg}, 68 \%$ ) as a yellow foam.

32: $R_{\mathrm{f}}=0.55$ (silica gel, $\left.\mathrm{CH}_{2} \mathrm{Cl}_{2}: \mathrm{MeOH}, 93: 7\right) ;[\alpha]_{\mathrm{D}}^{32}=-55.2\left(\mathrm{CHCl}_{3}, c=1.00\right)$; IR (film) $v_{\max }$ 3395, 3315, 2928, 2843, 1657, 1557, 1527, 1478, 1378, 1246, 1213, 1092, 835, $741 \mathrm{~cm}^{-1} ;{ }^{1} \mathrm{H}$ NMR $\left(600 \mathrm{MHz}, \mathrm{CD}_{3} \mathrm{CN}, 70{ }^{\circ} \mathrm{C}\right) \delta 8.15(\mathrm{~s}, 1 \mathrm{H}), 8.09(\mathrm{~s}, 1 \mathrm{H}), 8.02(\mathrm{~d}, J=7.9 \mathrm{~Hz}, 1 \mathrm{H}), 7.87(\mathrm{~d}$, 
$J=8.3 \mathrm{~Hz}, 1 \mathrm{H}), 7.84-7.82(\mathrm{~m}, 2 \mathrm{H}), 7.76(\mathrm{~d}, J=7.9 \mathrm{~Hz}, 1 \mathrm{H}), 7.73(\mathrm{~d}, J=7.0 \mathrm{~Hz}, 1 \mathrm{H}), 7.57-$ 7.56 (m, 2 H), $7.50-7.48$ (m, 2 H), 7.46 (br s, 1 H), $7.41-7.38$ (m, 2 H), 7.32 (t, J= 7.4 Hz, 2 H), $7.26-7.15(\mathrm{~m}, 9 \mathrm{H}), 7.14(\mathrm{~d}, J=4.0 \mathrm{~Hz}, 1 \mathrm{H}), 6.87(\mathrm{~s}, 1 \mathrm{H}), 6.86(\mathrm{~d}, J=10.1 \mathrm{~Hz}, 1 \mathrm{H}), 6.36$ $(\mathrm{dd}, J=10.1,4.0 \mathrm{~Hz}, 1 \mathrm{H}), 5.56(\mathrm{~d}, J=9.7 \mathrm{~Hz}, 1 \mathrm{H}), 5.37(\mathrm{~s}, 1 \mathrm{H}), 5.25-5.23(\mathrm{~m}, 2 \mathrm{H}), 4.97$ (m, $1 \mathrm{H}), 4.84-4.82(\mathrm{~m}, 1 \mathrm{H}), 4.77(\mathrm{~d}, J=3.5 \mathrm{~Hz}, 1 \mathrm{H}), 4.72-4.60(\mathrm{~m}, 5 \mathrm{H}), 4.52-4.49$ (m, $2 \mathrm{H})$, $4.41(\mathrm{t}, J=6.0 \mathrm{~Hz}, 1 \mathrm{H}), 4.38-4.31(\mathrm{~m}, 2 \mathrm{H}), 4.28-4.24(\mathrm{~m}, 2 \mathrm{H}), 4.19(\mathrm{t}, J=8.2 \mathrm{~Hz}, 1 \mathrm{H})$, $4.10-4.05(\mathrm{~m}, 2 \mathrm{H}), 3.63$ - $3.55(\mathrm{~m}, 4 \mathrm{H}), 3.48$ - $3.45(\mathrm{~m}, 1 \mathrm{H}), 3.44-3.32$ (m, $4 \mathrm{H}), 3.30$ 3.10 (m, 2 H), $2.96-2.86$ (br, 1 H), 2.45 (br s, 1 H), 1.35 (d, $J=7.0$ Hz, 3 H), 1.32 (d, $J=6.1$ $\mathrm{Hz}, 3 \mathrm{H}), 1.24-1.21(\mathrm{~m}, 9 \mathrm{H}), 1.16(\mathrm{~d}, J=6.1 \mathrm{~Hz}, 3 \mathrm{H}), 1.09(\mathrm{~d}, J=6.1 \mathrm{~Hz}, 3 \mathrm{H}), 1.03(\mathrm{~s}, 3 \mathrm{H})$, $0.97-0.90(\mathrm{~m}, 27 \mathrm{H}), 0.88(\mathrm{~s}, 9 \mathrm{H}), 0.82(\mathrm{~d}, J=6.6 \mathrm{~Hz}, 3 \mathrm{H}), 0.75(\mathrm{~s}, 9 \mathrm{H}), 0.66(\mathrm{q}, J=7.9 \mathrm{~Hz}$, $6 \mathrm{H}), 0.17(\mathrm{~s}, 3 \mathrm{H}), 0.16(\mathrm{~s}, 3 \mathrm{H}), 0.11(\mathrm{~s}, 3 \mathrm{H}), 0.10$ (s, $3 \mathrm{H}), 0.09$ (s, $3 \mathrm{H}), 0.08$ (s, $3 \mathrm{H}),-0.03$ $(\mathrm{s}, 3 \mathrm{H}),-0.11(\mathrm{~s}, 3 \mathrm{H}) ;{ }^{13} \mathrm{C}$ NMR $\left(150 \mathrm{MHz}\right.$, dioxane- $\left.d_{8}, 70{ }^{\circ} \mathrm{C}\right) \delta 174.6,173.6,173.1,172.7$, 172.2 , 171.1, 170.7, 170.6, 169.8, 169.3, 165.6, 161.1, 160.0, 151.5, 151.3, 149.8, 147.1, 145.3, $145.2,142.2,142.2,133.5,133.4,133.2,132.8,132.8,132.2,131.2,131.0,130.7,130.5,130.5$, $129.9,129.8,129.8,129.9,129.0,128.3,127.8,127.7,127.7,127.6,127.3,126.2$, 126.1, 122.6, $122.3,120.6,120.6,75.5,71.3,68.0,67.8,64.9,58.2,53.9,53.8,50.5,47.9,39.3,38.7,34.0$, $32.5,30.4,30.3,30.2,30.1,30.0,29.9,29.8,29.7,29.6,27.8,26.5,26.3,26.2,26.0,25.4,24.9$, 23.2, 18.8, 18.6, 17.9, 16.2, 14.2, 11.7, 7.1, 5.6, -4.0, -4.3, -4.7, -4.8; HRMS (ESI-TOF) calcd for $\mathrm{C}_{134} \mathrm{H}_{187} \mathrm{~N}_{19} \mathrm{O}_{20} \mathrm{~S}_{5} \mathrm{Se}_{3} \mathrm{Si}_{5}{ }^{+}\left[\mathrm{M}+\mathrm{H}^{+}\right]$2922.9217, found 2922.9215.

\section{Triene seco ester 33 (Scheme 7):}

To seco ester $32(3.0 \mathrm{mg}, 0.0010 \mathrm{mmol})$ in $\mathrm{CH}_{2} \mathrm{Cl}_{2}(0.10 \mathrm{~mL})$ at $0{ }^{\circ} \mathrm{C}$ was added $t$ - $\mathrm{BuOOH}(60$ $\mu \mathrm{L}$ of $5-6 \mathrm{M}$ decane solution). The reaction mixture was stirred for $5 \mathrm{~min}$ at $0{ }^{\circ} \mathrm{C}$ and then warmed to $25^{\circ} \mathrm{C}$. Stirring was continued for $20 \mathrm{~min}$, and then a further $60 \mu \mathrm{L}$ of the above $t$ $\mathrm{BuOOH}$ solution was added. The resulting mixture was stirred for a further $30 \mathrm{~min}$, cooled to 0 ${ }^{\circ} \mathrm{C}$, quenched with a saturated aqueous $1: 1$ solution of $\mathrm{NaHCO}_{3}$ and $\mathrm{Na}_{2} \mathrm{~S}_{2} \mathrm{O}_{3}(2 \mathrm{~mL})$, and then warmed to $25{ }^{\circ} \mathrm{C}$ and stirred for $10 \mathrm{~min}$. The aqueous layer was extracted with $\mathrm{CH}_{2} \mathrm{Cl}_{2}(3 \times 2$ $\mathrm{mL})$ then dried $\left(\mathrm{Na}_{2} \mathrm{SO}_{4}\right)$ and concentrated. The crude residue was purified by preparative TLC (silica gel, $\left.\mathrm{CH}_{2} \mathrm{Cl}_{2}: \mathrm{MeOH}, 92: 8\right)$ to give triene seco ester 33 (1.9 mg, 74\%) as a yellow foam.

33: $R_{\mathrm{f}}=0.42$ (silica gel, $\left.\mathrm{CH}_{2} \mathrm{Cl}_{2}: \mathrm{MeOH}, 93: 7\right)$; $[\alpha]_{\mathrm{D}}{ }^{32}=-19.5\left(\mathrm{CHCl}_{3}, c=0.15\right)$; IR (film) $v_{\max }$ $3352,2919,2850,1702,1655,1521,1464,1378,1251,1212,1099,835,741 \mathrm{~cm}^{-1}$; ${ }^{1} \mathrm{H}$ NMR 
$\left(600 \mathrm{MHz}, \mathrm{CD}_{3} \mathrm{CN}, 70{ }^{\circ} \mathrm{C}\right) \delta 9.77(\mathrm{~s}, 1 \mathrm{H}), 8.90(\mathrm{~s}, 1 \mathrm{H}), 8.26(\mathrm{~s}, 1 \mathrm{H}), 8.14(\mathrm{~s}, 1 \mathrm{H}), 8.09(\mathrm{~s}, 1$ H), $7.91(\mathrm{~d}, J=8.3 \mathrm{~Hz}, 1 \mathrm{H}), 7.83(\mathrm{dd}, J=7.9,4.4 \mathrm{~Hz}, 2 \mathrm{H}), 7.77-7.71(\mathrm{~m}, 3 \mathrm{H}), 7.65-7.61$ (br, $1 \mathrm{H}), 7.58$ (br s, $1 \mathrm{H}), 7.41$ (q, $J=7.5 \mathrm{~Hz}, 2 \mathrm{H}), 7.32$ (t, $J=7.3 \mathrm{~Hz}, 3 \mathrm{H}), 7.22$ (d, $J=8.1 \mathrm{~Hz}$,

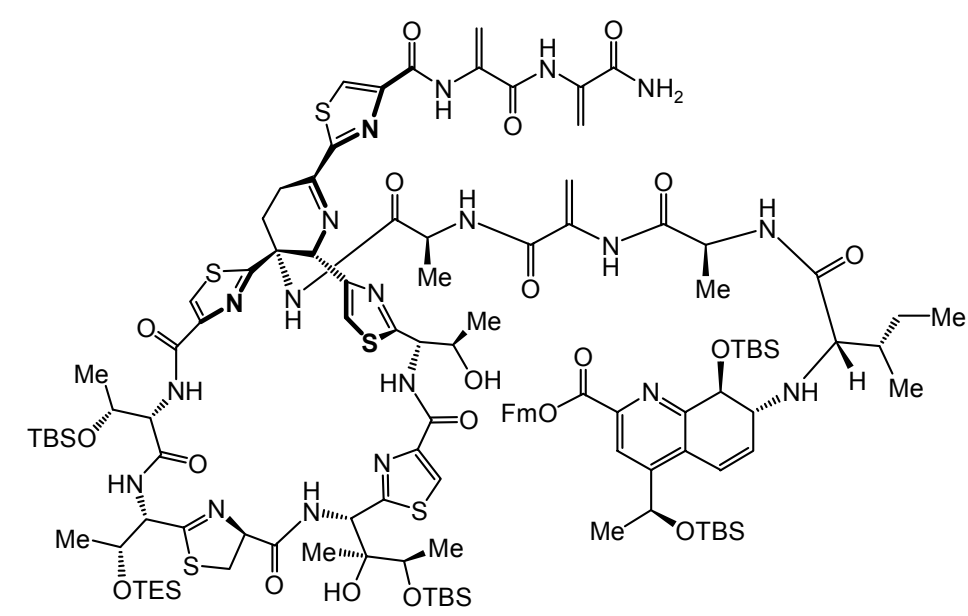

33

$1 \mathrm{H}), 6.87(\mathrm{~d}, J=10.0 \mathrm{~Hz}, 1 \mathrm{H}), 6.51$ (d, $J=1.8 \mathrm{~Hz}, 1 \mathrm{H}), 6.39-6.34(\mathrm{~m}$, $1 \mathrm{H}), 6.37(\mathrm{~d}, J=1.7 \mathrm{~Hz}, 1 \mathrm{H}), 5.95$ (s, $1 \mathrm{H}), 5.59-5.52(\mathrm{~m}, 3 \mathrm{H}), 5.46$ (s, $1 \mathrm{H}), 5.39(\mathrm{~s}, 1 \mathrm{H}), 5.36(\mathrm{t}, J=5.3$ $\mathrm{Hz}, 1 \mathrm{H}), 5.26(\mathrm{q}, J=6.6 \mathrm{~Hz}, 1 \mathrm{H})$, $5.19-5.13(\mathrm{~m}, 1 \mathrm{H}), 4.95(\mathrm{dt}, J=$ 9.2, $1.7 \mathrm{~Hz}, 1 \mathrm{H}), 4.85-4.81(\mathrm{~m}, 1$ H), $4.75-4.73$ (m, $1 \mathrm{H}), 4.72$ (dd, $J$

$=6.6,4.0 \mathrm{~Hz}, 1 \mathrm{H}), 4.66(\mathrm{dd}, J=$

10.9, 7.0 Hz, $1 \mathrm{H}), 4.52-4.49(\mathrm{~m}, 2 \mathrm{H}), 4.40(\mathrm{t}, J=7.0 \mathrm{~Hz}, 1 \mathrm{H}), 4.27-4.20(\mathrm{~m}, 3 \mathrm{H}), 4.07$ (q, $J$ $=7.0 \mathrm{~Hz}, 1 \mathrm{H}), 3.29-3.21(\mathrm{~m}, 2 \mathrm{H}), 2.98-2.92(\mathrm{br}, 1 \mathrm{H}), 2.52-2.44(\mathrm{br}, 1 \mathrm{H}), 1.35(\mathrm{~d}, J=6.0$ $\mathrm{Hz}, 3 \mathrm{H}), 1.32(\mathrm{~d}, J=7.0 \mathrm{~Hz}, 3 \mathrm{H}), 1.26-1.22(\mathrm{~m}, 9 \mathrm{H}), 1.17$ (d, $J=7.0 \mathrm{~Hz}, 3 \mathrm{H}), 1.09$ (d, $J=$ $6.0 \mathrm{~Hz}, 3 \mathrm{H}), 1.01$ (s, $3 \mathrm{H}), 0.96-0.89$ (m, $36 \mathrm{H}), 0.82$ (d, $J=6.6 \mathrm{~Hz}, 3 \mathrm{H}), 0.74$ (s, 9 H), 0.63 (q, J= $7.9 \mathrm{~Hz}, 6 \mathrm{H}), 0.18(\mathrm{~s}, 3 \mathrm{H}), 0.17(\mathrm{~s}, 3 \mathrm{H}), 0.11(\mathrm{~s}, 3 \mathrm{H}), 0.10(\mathrm{~s}, 3 \mathrm{H}), 0.09$ (s, $3 \mathrm{H}), 0.08$ (s, $3 \mathrm{H}),-0.02(\mathrm{~s}, 3 \mathrm{H}),-0.13(\mathrm{~s}, 3 \mathrm{H}) ;{ }^{13} \mathrm{C}$ NMR $\left(150 \mathrm{MHz}\right.$, dioxane- $\left.d_{8}, 70{ }^{\circ} \mathrm{C}\right) \delta 166.2,165.3$, 162.5, 162.4, 156.2, 144.4, 140.8, 133.6, 128.1, 126.3, 124.5, 119.1, 76.5, 69.3, 67.4, 66.5, 64.7, 56.6, 52.0, 49.3, 45.7, 40.3, 38.5, 37.5, 33.0, 29.4, 28.5, 25.8, 24.9, 17.6, 5.8, -3.2, -5.9, -7.2; HRMS (ESI-TOF) calcd for $\mathrm{C}_{116} \mathrm{H}_{170} \mathrm{~N}_{19} \mathrm{O}_{20} \mathrm{~S}_{5} \mathrm{Si}_{5}{ }^{+}\left[\mathrm{M}+\mathrm{H}^{+}\right]$2449.0313, found 2449.0309.

\section{Truncated hydroxy acid 34 (Scheme 7):}

To triene seco ester $33(1.9 \mathrm{mg}, 0.00078 \mathrm{mmol})$ at $0{ }^{\circ} \mathrm{C}$ was added $0.10 \mathrm{~mL}$ of a $10: 1$ solution of $\mathrm{CH}_{2} \mathrm{Cl}_{2}: \mathrm{Et}_{2} \mathrm{NH}$. The reaction mixture was stirred for $5 \mathrm{~min}$ at $0{ }^{\circ} \mathrm{C}$ and for a further $2.5 \mathrm{~h}$ at 25 ${ }^{\circ} \mathrm{C}$, then diluted with toluene $(3 \mathrm{~mL})$ and concentrated. The crude residue was purified by preparative TLC (silica gel, $\mathrm{CH}_{2} \mathrm{Cl}_{2}: \mathrm{MeOH}, 92: 8$ ) to give truncated hydroxy acid 34 (1.3 mg, $78 \%)$ as a yellow oil. 


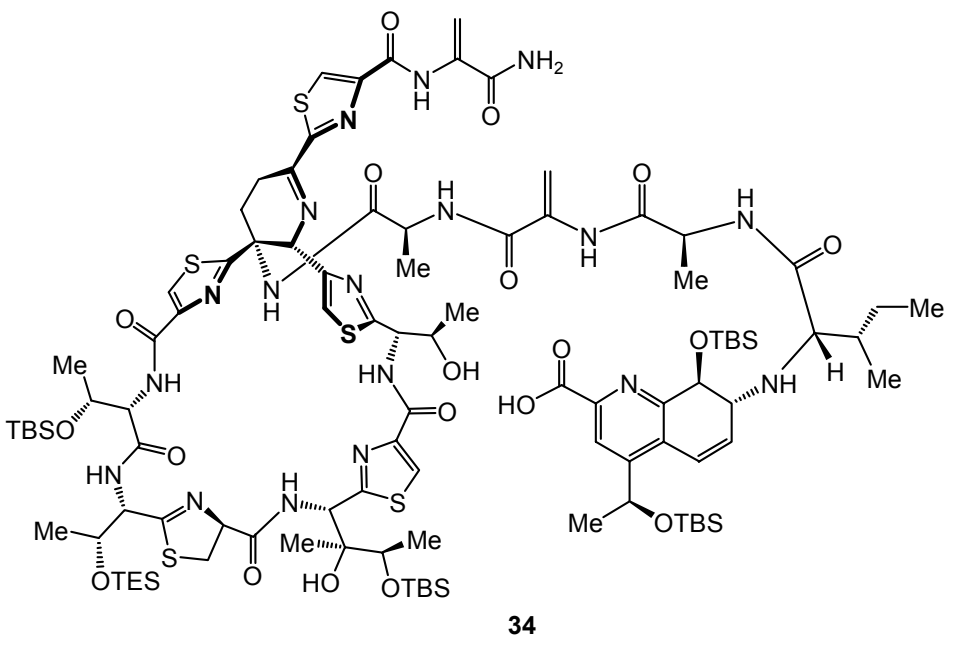

34: $\quad R_{\mathrm{f}}=0.23 \quad$ (silica gel, $\left.\mathrm{CH}_{2} \mathrm{Cl}_{2}: \mathrm{MeOH}, \quad 93: 7\right) ; \quad[\alpha]_{\mathrm{D}}^{32}=$ $-22.4\left(\mathrm{CHCl}_{3}, c=0.12\right)$; IR (film) $v_{\max } 3358,2931,2864,1709,1654$, $1521,1458,1386,1254,1212,1097$, $831,741 \mathrm{~cm}^{-1} ;{ }^{1} \mathrm{H}$ NMR $(500 \mathrm{MHz}$, $\left.\mathrm{CD}_{3} \mathrm{CN}, 70{ }^{\circ} \mathrm{C}\right) \delta 9.88(\mathrm{~s}, 1 \mathrm{H}), 8.26$ (s, $1 \mathrm{H}), 8.20$ (s, $1 \mathrm{H}), 8.15(\mathrm{~s}, 1 \mathrm{H})$, $7.95(\mathrm{~d}, J=8.1 \mathrm{~Hz}, 1 \mathrm{H}), 7.81-7.78$ (br, $1 \mathrm{H}), 7.61-7.56$ (br, $1 \mathrm{H}), 7.49$ (s, $1 \mathrm{H}), 7.29-7.22(\mathrm{~m}, 2 \mathrm{H}), 6.90(\mathrm{~d}, J=9.9 \mathrm{~Hz}, 1 \mathrm{H}), 6.55(\mathrm{~d}, J=1.5 \mathrm{~Hz}, 1 \mathrm{H}), 6.38-6.34(\mathrm{~m}$, $1 \mathrm{H}), 5.99(\mathrm{~s}, 1 \mathrm{H}), 5.59-5.52(\mathrm{~m}, 3 \mathrm{H}), 5.41(\mathrm{~s}, 1 \mathrm{H}), 5.37(\mathrm{t}, J=5.7 \mathrm{~Hz}, 1 \mathrm{H}), 5.29(\mathrm{q}, J=6.2$ $\mathrm{Hz}, 1 \mathrm{H}), 5.21-5.18(\mathrm{~m}, 1 \mathrm{H}), 4.96(\mathrm{~d}, J=9.3 \mathrm{~Hz}, 1 \mathrm{H}), 4.85-4.81(\mathrm{~m}, 1 \mathrm{H}), 4.76(\mathrm{~s}, 1 \mathrm{H}), 4.56$ - 4.51 (m, 2 H), $4.41-4.37$ (m, 1 H), 4.31 - 4.27 (m, 3 H), 4.11 - 4.06 (m, 1 H), 3.46 - 3.44 (m, $1 \mathrm{H}), 3.29-3.21$ (m, $2 \mathrm{H}), 2.98-2.92$ (br, $1 \mathrm{H}), 2.52-2.44$ (br, $1 \mathrm{H}), 1.38$ (d, $J=6.6 \mathrm{~Hz}, 3 \mathrm{H})$, $1.36(\mathrm{~d}, J=7.0 \mathrm{~Hz}, 3 \mathrm{H}), 1.26(\mathrm{~d}, J=6.2 \mathrm{~Hz}, 3 \mathrm{H}), 1.20$ (d, $J=6.3 \mathrm{~Hz}, 3 \mathrm{H}), 1.13-1.08$ (m, 9 H), 1.03 (s, 3 H), $0.99-0.89$ (m, 36 H), 0.86 (d, $J=6.6 \mathrm{~Hz}, 3 \mathrm{H}), 0.72$ (s, 9 H), 0.63 (q, $J=7.9$ Hz, 6 H), 0.19 (s, 3 H), 0.18 (s, 3 H), 0.11 (s, 3 H), 0.10 (s, 3 H), 0.09 (s, 3 H), 0.08 (s, 3 H), $-0.01(\mathrm{~s}, 3 \mathrm{H}),-0.12(\mathrm{~s}, 3 \mathrm{H})$; HRMS (ESI-TOF) calcd for $\mathrm{C}_{99} \mathrm{H}_{157} \mathrm{~N}_{18} \mathrm{O}_{19} \mathrm{~S}_{5} \mathrm{Si}_{5}{ }^{+}\left[\mathrm{M}+\mathrm{H}^{+}\right]$ 2201.9322 , found 2201.9283 .

\section{Truncated dehydroalanine 35 (Scheme 8):}

To bis-dehydroalanine model system $24(20 \mathrm{mg}, 0.040 \mathrm{mmol})$ at $0{ }^{\circ} \mathrm{C}$ was added $1.0 \mathrm{~mL}$ of a 6.3:1 solution of $\mathrm{CH}_{2} \mathrm{Cl}_{2}: \mathrm{Et}_{2} \mathrm{NH}$. The reaction mixture was stirred for $30 \mathrm{~min}$ at $0{ }^{\circ} \mathrm{C}$ and $2 \mathrm{~h}$ at $25^{\circ} \mathrm{C}$, then diluted in EtOAc $(10 \mathrm{~mL})$ and washed with aqueous $5 \%$

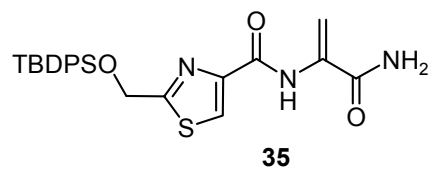

$\mathrm{HCl}$ solution, saturated aqueous $\mathrm{NaHCO}_{3}$ solution, and brine $(5 \mathrm{~mL}$ each), then dried $\left(\mathrm{Na}_{2} \mathrm{SO}_{4}\right)$ and concentrated. The residue was purified by flash column chromatography (silica gel, hexanes:EtOAc, 1:2) to give truncated dehydroalanine $35(16.1 \mathrm{mg}, 93 \%)$ as a white powder.

35: $R_{\mathrm{f}}=0.68$ (silica gel, hexanes:EtOAc, 3:7); IR (film) $v_{\max } 3366,2931,2847,1658,1523$, 1486, 1192, $743 \mathrm{~cm}^{-1} ;{ }^{1} \mathrm{H}$ NMR (500 MHz, $\left.\mathrm{CDCl}_{3}\right) \delta 9.78$ (br s, $\left.1 \mathrm{H}\right), 8.13$ (s, $\left.1 \mathrm{H}\right), 7.71-7.68$ 
(m, $4 \mathrm{H}), 7.49-7.40(\mathrm{~m}, 6 \mathrm{H}), 6.68(\mathrm{~d}, J=2.2 \mathrm{~Hz}, 1 \mathrm{H}), 6.08(\mathrm{br}, 1 \mathrm{H}), 5.75(\mathrm{br}, 1 \mathrm{H}), 5.37$ (t, $J$ $=1.5 \mathrm{~Hz}, 1 \mathrm{H}), 4.99(\mathrm{~s}, 2 \mathrm{H}), 1.15(\mathrm{~s}, 9 \mathrm{H}) ;{ }^{13} \mathrm{C} \mathrm{NMR}\left(150 \mathrm{MHz}, \mathrm{CDCl}_{3}\right) \delta$ 173.3, 165.9, 160.1, 150.0, 135.7, 133.9, 132.5, 130.3, 128.2, 123.8, 103.2, 64.0, 26.9, 19.5; HRMS (ESI-TOF) calcd for $\mathrm{C}_{24} \mathrm{H}_{28} \mathrm{~N}_{3} \mathrm{O}_{3} \mathrm{SSi}^{+}\left[\mathrm{M}+\mathrm{H}^{+}\right]$465.1615, found 465.1619.

\section{Macrolide triene 46 (Scheme 9):}

To seco ester $32(31 \mathrm{mg}, 0.011 \mathrm{mmol})$ at $0{ }^{\circ} \mathrm{C}$ was added $4 \mathrm{~mL}$ of a $6.5: 1$ solution of $\mathrm{CH}_{2} \mathrm{Cl}_{2}: \mathrm{Et}_{2} \mathrm{NH}$, and the resulting mixture stirred for $5 \mathrm{~min}$ at $0{ }^{\circ} \mathrm{C}$ and then warmed to $25{ }^{\circ} \mathrm{C}$.

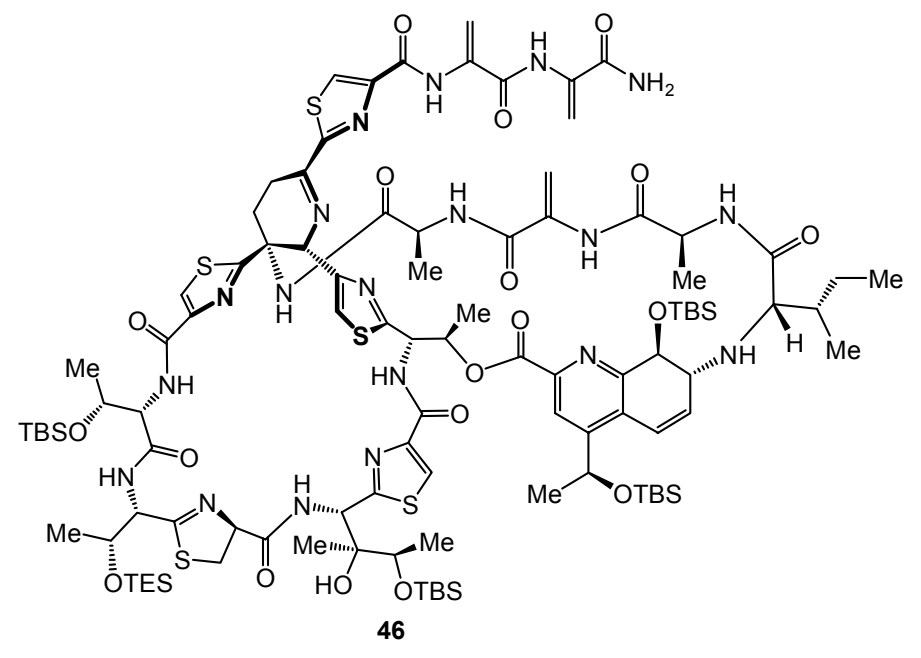

After stirring for $2.5 \mathrm{~h}$ at $25^{\circ} \mathrm{C}$, toluene was added and the reaction mixture was concentrated. The residue was purified by preparative TLC (silica gel, $\left.\mathrm{CH}_{2} \mathrm{Cl}_{2}: \mathrm{MeOH}, 92.5: 7.5\right)$ to give carboxylic acid $44(25 \mathrm{mg}, 87 \%)$ as a tan foam. To carboxylic acid $44(25 \mathrm{mg}$, $0.0091 \mathrm{mmol})$ in toluene $(0.50 \mathrm{~mL})$ at 25 ${ }^{\circ} \mathrm{C}$ was added triethylamine $(51 \mu \mathrm{L}, 0.36$ mmol) and 2,4,6-trichlorobenzoyl

chloride $(0.043 \mathrm{~mL}, 0.27 \mathrm{mmol})$. The reaction mixture was stirred for $24 \mathrm{~h}$ at $25^{\circ} \mathrm{C}$, then diluted with toluene (total volume $=4.25 \mathrm{~mL}$ ) and added dropwise over the course of $12 \mathrm{~h}$ (syringe pump) to a solution of DMAP (33 $\mathrm{mg}, 0.27 \mathrm{mmol})$ in toluene $(18.3 \mathrm{~mL})$. After addition was complete, the resulting mixture was stirred at $25{ }^{\circ} \mathrm{C}$ for a further $12 \mathrm{~h}$, then cooled to $0{ }^{\circ} \mathrm{C}$ and acidified to $\mathrm{pH} \sim 3$ with an aqueous $10 \mathrm{mg} / \mathrm{mL}$ solution of $\mathrm{KHSO}_{4}$. The layers were separated and the aqueous layer was re-extracted with EtOAc $(2 \times 10 \mathrm{~mL})$. The combined organic layers were then washed with a $1: 1$ solution of saturated aqueous $\mathrm{NaHCO}_{3}$ :brine $(10 \mathrm{~mL})$ and the aqueous layer was re-extracted with EtOAc $(2 \times 10 \mathrm{~mL})$. The combined organic layers were dried $\left(\mathrm{Na}_{2} \mathrm{SO}_{4}\right)$ and concentrated. The residue was purified by preparative TLC (silica gel, $\left.\mathrm{CH}_{2} \mathrm{Cl}_{2}: \mathrm{MeOH}, 93: 7\right)$ to give macrolide 45 as a pale yellow foam (10.7 mg, $42 \%$ ). (Note: due to low solubility and extremely restricted rotation of the bulky phenylselenium and silyl protecting groups, no meaningful spectral data could be acquired for $\mathbf{4 5}$.) To a suspension of macrolide $\mathbf{4 5}$ (10.7 mg, $0.0039 \mathrm{mmol})$ and $\mathrm{NaHCO}_{3}(10.7 \mathrm{mg}, 0.13 \mathrm{mmol})$ in $\mathrm{CH}_{2} \mathrm{Cl}_{2}(1.2 \mathrm{~mL})$ at $0{ }^{\circ} \mathrm{C}$ was 
added $t$ - $\mathrm{BuOOH}(0.13 \mathrm{~mL}$ of a $5-6 \mathrm{M}$ solution in decane). The reaction mixture was stirred for $5 \mathrm{~min}$ at $0{ }^{\circ} \mathrm{C}$ and then warmed to $25{ }^{\circ} \mathrm{C}$. Stirring was continued for $1 \mathrm{~h}$ at $25{ }^{\circ} \mathrm{C}$, then a further $0.13 \mathrm{~mL}$ of the $5-6 \mathrm{M}$ decane solution of $t$ - $\mathrm{BuOOH}$ was added. Stirring was continued for $1 \mathrm{~h}$, then the reaction mixture was cooled to $0{ }^{\circ} \mathrm{C}$, and EtOAc $(12 \mathrm{~mL})$ and a saturated aqueous $1: 1$ solution of $\mathrm{Na}_{2} \mathrm{~S}_{2} \mathrm{O}_{3}$ and $\mathrm{NaHCO}_{3}(7 \mathrm{~mL})$ were added. The layers were separated and the aqueous phase was re-extracted with EtOAc $(2 \times 5 \mathrm{~mL})$. The combined organic layers were dried $\left(\mathrm{Na}_{2} \mathrm{SO}_{4}\right)$ and concentrated. The residue was purified by preparative TLC (silica gel, $\left.\mathrm{CH}_{2} \mathrm{Cl}_{2}: \mathrm{MeOH}, 93: 7\right)$ to give macrolide triene 46 (5.9 mg, 68\%) as a pale yellow foam.

46: $R_{\mathrm{f}}=0.38$ (silica gel, $\left.\mathrm{CHCl}_{3}: \mathrm{MeOH}, 92: 8\right) ;[\alpha]_{\mathrm{D}}^{32}=-23.6\left(\mathrm{CHCl}_{3}, c=0.19\right)$; IR (film) $v_{\max }$ 3354, 2924, 2852, 1705, 1658, 1501, 1464, 1381, 1250, 1219, 1098, 832, $779 \mathrm{~cm}^{-1}$; ${ }^{1} \mathrm{H}$ NMR $\left(600 \mathrm{MHz}, \mathrm{CD}_{3} \mathrm{CN}, 70{ }^{\circ} \mathrm{C}\right) \delta 9.72(\mathrm{~s}, 1 \mathrm{H}), 8.88(\mathrm{~s}, 1 \mathrm{H}), 8.25$ (br s, $\left.1 \mathrm{H}\right), 8.24(\mathrm{~s}, 1 \mathrm{H}), 7.99$ (br s, 1 H), 7.49 (br s, 1H), 7.34 (br s, 1 H), 7.12 (d, J=4.3 Hz, 1 H), 6.90 (d, J=9.6 Hz, 1 H), 6.50 $(\mathrm{d}, J=1.0 \mathrm{~Hz}, 1 \mathrm{H}), 6.34(\mathrm{~d}, J=1.0 \mathrm{~Hz}, 1 \mathrm{H}), 6.31-6.29(\mathrm{~m}, 1 \mathrm{H}), 5.85$ (br s, $1 \mathrm{H}), 5.57(\mathrm{~s}, 1$ $\mathrm{H}), 5.55(\mathrm{~s}, 1 \mathrm{H}), 5.48(\mathrm{t}, J=7.1 \mathrm{~Hz}, 1 \mathrm{H}), 5.42(\mathrm{~d}, J=7.7 \mathrm{~Hz}, 1 \mathrm{H}), 5.36(\mathrm{~s}, 1 \mathrm{H}), 5.21(\mathrm{~d}, J=$ $6.4 \mathrm{~Hz}, 2 \mathrm{H}), 4.89-4.82(\mathrm{~m}, 2 \mathrm{H}), 4.71$ (s, $1 \mathrm{H}), 4.56$ (br s, $1 \mathrm{H}), 4.39-4.22$ (m, 4 H), 4.01 (d, $=6.8 \mathrm{~Hz}, 1 \mathrm{H}), 3.68(\mathrm{t}, J=7.4 \mathrm{~Hz}, 1 \mathrm{H}), 3.42-3.39(\mathrm{~m}, 3 \mathrm{H}), 3.04(\mathrm{~d}, J=5.7 \mathrm{~Hz}, 1 \mathrm{H}), 2.53-$ 2.48 (br, $1 \mathrm{H}), 2.42-2.38$ (br, $1 \mathrm{H}), 1.36$ (d, $J=6.6 \mathrm{~Hz}, 3 \mathrm{H}), 1.26$ (d, $J=6.1 \mathrm{~Hz}, 3 \mathrm{H}), 1.23-$ $1.20(\mathrm{~m}, 9 \mathrm{H}), 1.16-1.13(\mathrm{~m}, 9 \mathrm{H}), 1.10(\mathrm{~d}, J=7.1 \mathrm{~Hz}, 3 \mathrm{H}), 0.92-0.88$ (m, $36 \mathrm{H}), 0.74(\mathrm{~s}, 9$ H), 0.63 (q, $J=7.9 \mathrm{~Hz}, 6 \mathrm{H}), 0.18$ (s, $3 \mathrm{H}), 0.17$ (s, $3 \mathrm{H}), 0.14$ (s, $3 \mathrm{H}), 0.10$ (s, $3 \mathrm{H}), 0.09$ (s, 3 $\mathrm{H}), 0.08(\mathrm{~s}, 3 \mathrm{H}), 0.06(\mathrm{~s}, 3 \mathrm{H}),-0.05(\mathrm{~s}, 3 \mathrm{H}),-0.18(\mathrm{~s}, 3 \mathrm{H}) ;{ }^{13} \mathrm{C}$ NMR $\left(150 \mathrm{MHz}\right.$, dioxane- $d_{8}$, $\left.70{ }^{\circ} \mathrm{C}\right) \delta 172.4,171.9,171.0,169.4,168.9,168.6,167.8,166.9,166.8,165.3,163.7,161.5$, $160.5,160.1,159.5,159.3,159.2,157.1,156.2$, 153.0, 151.8, 149.0, 148.9, 145.1, 142.0, 145.2, $133.2,132.5,131.4,129.2,127.9,127.8,127.6,125.4,125.3,122.9,122.7,121.2,120.5,115.7$, $100.0,99.9,77.8,75.9,70.8,68.6,58.1,55.9,54.3,53.8,50.1,47.2,37.0,31.4,29.9,27.5,27.2$, 27.1, 25.1, 24.0, 22.9, 20.9, 20.5, 17.8, 17.0, 16.8, 16.5, 15.5, 14.8, 13.2, 13.0, 11.6, 8.9, -1.4, 5.3; HRMS (ESI-TOF) calcd for $\mathrm{C}_{102} \mathrm{H}_{158} \mathrm{~N}_{19} \mathrm{O}_{19} \mathrm{~S}_{5} \mathrm{Si}_{5}{ }^{+}\left[\mathrm{M}+\mathrm{H}^{+}\right]$2252.9425, found 2252.9395. 


\section{Thiostrepton 1 (Scheme 9):}

To macrolide triene $46(5.9 \mathrm{mg}, 0.0027 \mathrm{mmol})$ at $0{ }^{\circ} \mathrm{C}$ was added a $4: 1$ solution of THF:HF $\bullet$ py $(6.0 \mathrm{~mL})$. The reaction mixture was stirred for $5 \mathrm{~min}$ at $0{ }^{\circ} \mathrm{C}$, and then for $24 \mathrm{~h}$ at $25{ }^{\circ} \mathrm{C}$. The

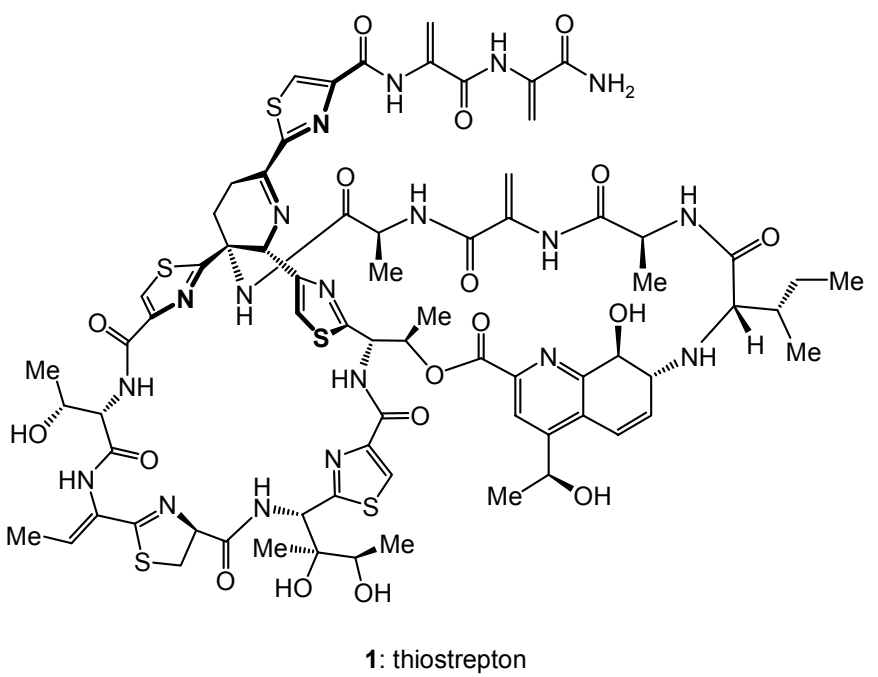

resulting mixture was then added to a saturated aqueous $\mathrm{NaHCO}_{3}$ solution (45 $\mathrm{mL})$ at $0{ }^{\circ} \mathrm{C}$ and the aqueous layer was extracted with $\mathrm{CHCl}_{3}(3 \times 25 \mathrm{~mL})$. The combined organic layers were dried $\left(\mathrm{Na}_{2} \mathrm{SO}_{4}\right)$ and concentrated. The residue was purified by preparative TLC (silica gel, $\left.\mathrm{CHCl}_{3}: \mathrm{MeOH}, 9: 1\right)$ to give synthetic thiostrepton $1(2.3 \mathrm{mg}, 52 \%)$ as a white powder.

1 (synthetic and natural): $R_{\mathrm{f}}=0.34$ (silica gel, $\left.\mathrm{CHCl}_{3}: \mathrm{MeOH}, 92: 8\right) ;[\alpha]_{\mathrm{D}}^{32}=-81.3\left(\mathrm{CHCl}_{3}, c=0.17\right)$ ); IR (film) $v_{\max } 3354,2978$, 2934, 1654, 1527, 1490, 1210, 1103, 1050, 938, 874, $755 \mathrm{~cm}^{-1}$; ${ }^{1} \mathrm{H}$ NMR (600 MHz, THF- $d_{8}$, thiostrepton is more soluble and stable in this solvent than in $\mathrm{CDCl}_{3}$; for proton numbering and abbreviations, see: ref $2 \mathrm{f}-\mathrm{h}$ in the previous article $\left.{ }^{1}\right): \delta 10.03(\mathrm{~s}, 1 \mathrm{H}, \mathrm{CONH}), 9.69(\mathrm{~s}, 1 \mathrm{H}$, CONH), 9.30 (s, $1 \mathrm{H}, \mathrm{CONH}$ ), 8.65 (s, $1 \mathrm{H}, \mathrm{CONH}$ ), 8.39 (s, $1 \mathrm{H}, \mathrm{Ar}-\mathrm{H}), 8.31$ (br s, $1 \mathrm{H}, \mathrm{OH}$ or $\mathrm{NH}), 8.29$ (s, $1 \mathrm{H}, \mathrm{Ar}-\mathrm{H}), 8.20$ (s, $1 \mathrm{H}, \mathrm{Ar}-\mathrm{H}), 8.03$ (br s, $1 \mathrm{H}, \mathrm{CONH}), 7.62$ (d, J=5.6 Hz, $1 \mathrm{H}$, CONH), 7.58 (d, $J=10.3 \mathrm{~Hz}, 1 \mathrm{H}, \mathrm{CONH}$ ), 7.54 (br s, $1 \mathrm{H}$, Thstn C3-OH), 7.53 (s, $1 \mathrm{H}, \mathrm{Ar}-\mathrm{H}$ ), 7.48 (br s, $1 \mathrm{H}, \mathrm{OH}$ or NH), 7.42 (d, $J=7.7 \mathrm{~Hz}, 1 \mathrm{H}, \mathrm{CONH}), 7.32$ (s, $1 \mathrm{H}, \mathrm{Ar}-\mathrm{H}), 7.07$ (d, $J=$ $7.7 \mathrm{~Hz}, 1 \mathrm{H}, \mathrm{QC} 8-\mathrm{OH}), 6.99$ (br s, $1 \mathrm{H}, \mathrm{OH}$ or NH), 6.92 (d, $J=9.7 \mathrm{~Hz}, \mathrm{Q} \mathrm{H}-5), 6.75$ (d, $J=2.0$ $\mathrm{Hz}, 1 \mathrm{H}$, Deala-H), 6.58 (d, $J=7.5 \mathrm{~Hz}, 1 \mathrm{H}, \mathrm{CONH}), 6.56$ (s, $1 \mathrm{H}$, Deala-H), 6.45 (q, $J=6.1 \mathrm{~Hz}$, $1 \mathrm{H}, \operatorname{Thr}(2) \mathrm{H}-3$ ), 6.32 (dd, $J=9.7,5.6 \mathrm{~Hz}, 1 \mathrm{H}, \mathrm{Q}$ H-6), 6.13 (q, $J=7.1 \mathrm{~Hz}, 1 \mathrm{H}$, But H-3), 5.89 (d, $J=9.8 \mathrm{~Hz}, 1 \mathrm{H}, \operatorname{Thr}(2) \mathrm{H}-2), 5.84$ (d, $J=9.2 \mathrm{~Hz}, 1 \mathrm{H}$, Thstn H-2), 5.77 (s, $1 \mathrm{H}$, Deala-H), 5.55 (s, 1 H, Deala-H), 5.49 (s, 1 H, OH or NH), $5.47-5.46$ (m, 1 H, Pip H-6ß), 5.30 (q, $J=6.1$ Hz, 1 H, Q H-11), 5.20 (s, 1 H, Deala-H), 5.04 (dd, $J=8.7,4.1$ Hz, 1 H, Cys H-4ß), 4.84 (t, $J=$ $7.7 \mathrm{~Hz}, 1 \mathrm{H}, \mathrm{Ala}(2) \mathrm{H}-2), 4.73$ (d, $J=7.1 \mathrm{~Hz}, 1 \mathrm{H}, \mathrm{Q} \mathrm{H}-8), 4.35$ (dd, $J=7.9,3.5 \mathrm{~Hz}, 1 \mathrm{H}, \operatorname{Thr}(1)$

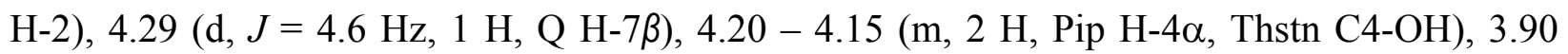
(quin, $J=6.5 \mathrm{~Hz}, 1 \mathrm{H}, \mathrm{Ala}(1) \mathrm{H}-2$ ), 3.85 (quin, $J=5.7 \mathrm{~Hz}, 1 \mathrm{H}$, Thstn $\mathrm{H}-4$ ), 3.24 (t, $J=12.3 \mathrm{~Hz}$, 
1 H, Cys H-5 $\alpha$ ), 3.00 - 2.93 (m, 2 H, Ile H-2, Pip H-3 $\alpha$ ), 2.39 - 2.35 (m, 1 H, Pip H-4 $\beta$ ), 1.60 $\left(\mathrm{d}, J=7.2 \mathrm{~Hz}, 3 \mathrm{H}, \mathrm{CH}_{3}\right), 1.33\left(\mathrm{~d}, J=3.5 \mathrm{~Hz}, 3 \mathrm{H}, \mathrm{CH}_{3}\right), 1.32\left(\mathrm{~d}, J=3.1 \mathrm{~Hz}, 3 \mathrm{H}, \mathrm{CH}_{3}\right), 1.27$ (d, $\left.J=6.6 \mathrm{~Hz}, 3 \mathrm{H}, \mathrm{CH}_{3}\right), 1.16\left(\mathrm{~s}, 3 \mathrm{H}, \mathrm{CH}_{3}\right), 1.14\left(\mathrm{~s}, 3 \mathrm{H}, \mathrm{CH}_{3}\right), 1.06\left(\mathrm{~d}, J=6.1 \mathrm{~Hz}, 3 \mathrm{H}, \mathrm{CH}_{3}\right), 0.96$ $\left(\mathrm{t}, J=7.0 \mathrm{~Hz}, 3 \mathrm{H}, \mathrm{CH}_{3}\right), 0.88\left(\mathrm{~d}, J=6.6 \mathrm{~Hz}, 3 \mathrm{H}, \mathrm{CH}_{3}\right) ;{ }^{13} \mathrm{C} \mathrm{NMR}\left(125 \mathrm{MHz}\right.$, THF- $\left.d_{8}\right) \delta 175.6$, 173.6, 172.0, 171.9, 171.3, 170.5, 170.2, 169.5, 168.3, 166.6, 166.3, 164.3, 162.8, 162.7, 162.3, $162.1,162.0,159.9,159.4,155.9,154.8,152.1,151.8,147.9,144.7,136.3,135.2,134.3,131.5$, 131.4, 130.6, 128.3, 125.6, 125.4, 123.7, 123.5, 118.4, 102.2, 101.8, 101.7, 80.8, 78.8, 73.7, 65.6, $65.2,61.1,58.8,57.3,56.7,54.2,52.7,50.0,39.9,35.8,30.7,27.1,24.0,20.9,19.8,19.6,19.4$, 18.1, 17.8, 16.1, 16.0, 12.0; HRMS (ESI-TOF) calcd for $\mathrm{C}_{72} \mathrm{H}_{86} \mathrm{~N}_{19} \mathrm{O}_{18} \mathrm{~S}_{5}^{+}\left[\mathrm{M}+\mathrm{H}^{+}\right]$1664.4996, found 1664.4976. 